\title{
The Hierarchy Problem and the Self-Localized Higgs
}

\author{
C.P. Burgess, ${ }^{1,2,3}$ Claudia de Rham ${ }^{1,2}$ and Leo van Nierop ${ }^{2}$ \\ ${ }^{1}$ Perimeter Institute for Theoretical Physics, Waterloo ON, N2L 2Y5, Canada. \\ 2 Physics 85 Astronomy, McMaster University, Hamilton ON, L8S 4M1, Canada. \\ 3 Theory Division, CERN, CH-1211 Geneva 23, Switzerland.
}

\begin{abstract}
We examine brane-world scenarios in which all the observed Standard Model particles reside on a brane but the Higgs is an elementary extra-dimensional scalar in the bulk. We show that, for codimension 2 branes, often-neglected interactions between the bulk Higgs and the branes cause two novel effects. First, they cause $\langle H\rangle$ to depend only logarithmically on the UV-sensitive coefficient, $m_{B}^{2}$, of the mass term, $m_{B}^{2} H^{*} H$, of the bulk potential, thus providing a new mechanism for tackling the hierarchy problem. Second, the Higgs brane couplings cause the lowest mass KK mode to localize near the brane without any need for geometrical effects like warping. We explore some preliminary implications such models have for the Higgs signature at the LHC, both in the case where the extra dimensions arise at the $\mathrm{TeV}$ scale, and in ADD models having Large Extra Dimensions. Novel Higgs features include couplings to fermions which can be different from Standard Model values, $m_{f} / v$, despite the fermions acquiring their mass completely from the Higgs expectation value.
\end{abstract}




\section{Contents}

1. Introduction 2

2. Vacuum Energetics of Extra-Dimensional Scalars 4

2.1 Codimension-one 4

2.2 Codimension-two 5

2.3 The Self-Localized State 9

3. A Self-Localized Bulk Higgs and the Hierarchy Problem 11

3.1 The Model 11

3.2 Scales and Naturalness 12

3.3 Higgs-induced Mass Terms 13

3.4 Couplings to the Higgs Fluctuations 16

4. Possible Signatures of a Bulk Higgs Scenario 17

4.1 Inclusive Processes 17

4.2 Higgs Decays to Fermions 19

4.3 TeV-Scale Compactifications 20

4.4 Large Extra Dimensions 22

5. Conclusions 22

A. Some Properties of Bessel Functions 26

B. Classical Divergences in Brane Couplings 27

C. Higher codimension 28

C.1 Coupling renormalization 28

C.2 Boundary condition and energy density 31

$\begin{array}{lll}\text { C.3 Codimension-3 } & 31\end{array}$

D. Bulk Goldstone modes $\quad 32$ 


\section{Introduction}

In the Standard Model (SM) the Higgs field is in many ways the odd man out. In the absence of the Higgs the only interactions that remain are gauge interactions, characterized by only a handful of coupling constants. But with the Higgs comes the deluge of parameters that parameterize our ignorance of the ultimate origins of the model's many masses and mixing angles. And among these parameters is the one dimensionful quantity, $\mu$, that governs the size of the $\mu^{2} H^{*} H$ term in the Higgs potential, and by fixing the size of the Higgs v.e.v. sets the scale for all masses. It is the sensitivity of this parameter to much heavier scales that is at the root of the hierarchy problem [1].

Historically, the hierarchy problem has been one of the main motivations for exploring brane-world scenarios for physics beyond the Standard Model [2, 3], for which all of the observed SM particles are trapped on a $(3+1)$-dimensional brane within an extra-dimensional bulk. Motivated by the observations that the Higgs is the lone SM particle yet to be observed, we here explore the idea that it is the only SM particle that is not confined to a brane: i.e. whereas all other SM particles live on a brane, the Higgs lives in the bulk. The hope is that this might account for its special role within the SM.

Brane-world models with the Higgs in the bulk have been examined in the literature, most often within the context of 5D Randall-Sundrum constructions [3]. Yet these models differ from the present proposal in one of two ways: either by imagining the extra-dimensional Higgs to be related to other fields by supersymmetry [4, 5]; or by taking the Higgs to be the $4 \mathrm{D}$ scalar component of what is 'really' an extra-dimensional gauge potential [6, 7]. The motivation for doing so is the expectation that the extra-dimensional gauge symmetries can help alleviate the hierarchy problem, potentially allowing some of the properties of Higgs interactions to be unified with those of the gauge interactions. Implicit in this is the belief that a Higgs that is a bona-fide extra-dimensional scalar makes no progress towards alleviating the hierarchy problems of the usual 4D Higgs.

A model more similar to the one studied here was considered in ref. [8, 9], although from a different point of view. In ref. [8] the authors study the effects of codimension-2 brane couplings on a massless bulk scalar, with a focus on couplings close to the critical value for which the symmetry-breaking properties of the vacuum change. Ref. [9] generalizes to massive bulk fields, but without the focus of this paper on the hierarchy problem, and consequently without the study of couplings to fermions and gauge bosons described herein.

It is simple to see why extra dimensions in themselves are generally believed not to alleviate the hierarchy problem. This is because the Higgs potential,

$$
U=-\frac{m_{B}^{2}}{2} H^{*} H+\frac{g}{4}\left(H^{*} H\right)^{2}
$$

is always minimized by $H^{*} H=m_{B}^{2} / g$, where in $n$ dimensions $g$ has the (engineering) di- 
mension of (mass $)^{4-n}$ while $m_{B}$ always simply has the dimension of mass. But the essence of the hierarchy problem is that because $m_{B}$ is proportional to a positive power of mass, it generically receives contributions from heavy particles that grow with the mass, $M$, of the particles involved, and so is dominantly affected by the heaviest such particle that can contribute. Since $m_{B}$ is a positive power of mass in any number of dimensions it is hard to see how the hierarchy problem can be ameliorated simply by placing the Higgs into the bulk.

In this paper we show why this simple argument is incorrect once the couplings between a bulk Higgs and the brane are properly taken into account. The brane-bulk interactions change the argument because the Higgs potential on the brane, $U_{b}$, and in the bulk, $U_{B}$, can disagree on which value of the Higgs v.e.v. has the least energy. In this case the system generically resolves this potential frustration by appropriately balancing these potential energies with the gradient energies which punish the field for attempting to interpolate between the two minima. But if the brane has codimension 2 (i.e. there are two dimensions transverse to the brane, such as for a (3+1)-dimensional brane situated in a $6 \mathrm{D}$ bulk), the Higgs likes to vary logarithmically near the branes, and the gradient energy associated with this variation is such that the resulting v.e.v. only depends logarithmically on the UV-sensitive term, $m_{B}$, of the bulk potential. Braneworld models can help with naturalness problems for a number of reasons; brane-bulk couplings provide a new way for them to do so. We show that the lunch is nevertheless not completely free, however, since the hierarchy problem gets partially recast as a requirement for the coefficients of the brane interactions $\left(H^{*} H\right)^{2}$ and $D_{M} H^{*} D^{M} H$ being required to be suppressed by very different scales. This kind of hierarchical suppression usually does not arise between two operators like these, that are not distinguished by lowenergy symmetries or selection rules.

We also show how Higgs-brane interactions change another fundamental piece of widelyheld intuition regarding the properties of a bulk Higgs. In the presence of a (positive) extradimensional mass term, $U_{B}=+\frac{1}{2} m_{B}^{2} H^{*} H$, the spectrum of Kaluza-Klein (KK) states would usually be expected to consist of a multitude of levels (generically spaced by $M_{c} \sim 2 \pi / L$ for a toroidal extra dimension of circumference $L$, say) that start at energies above a gap, $m_{k} \geq m_{B}$. We show here that brane-Higgs interactions can generically introduce a state which lives within this gap, $m<m_{B}$, that is 'bound' in the sense that its wave-function is localized at the position of the brane. We call this the 'self-localized' state inasmuch as its localization is a consequence only of the Higgs self-interactions and not on any geometric effects, such as those due to warping.

These arguments are presented in more detail in their simplest context in the next section, $\S 2$. $\S 3$ then tries to fashion an approach to the hierarchy problem by providing a preliminary discussion of the kinds of interactions that would be required for a realistic model, and the ways in which the low-energy Higgs couplings resemble and differ from those of the SM Higgs, as a function of the scales involved. $\S 4$ then follows with a discussion of some of the potential signatures and constraints such a scenario might have for Higgs physics. Our conclusions are briefly summarized in $\S 5$. 


\section{Vacuum Energetics of Extra-Dimensional Scalars}

In this section we describe the interplay between brane and bulk energetics for the simplest toy model: a single real scalar, $\phi$, in the presence of both brane and bulk potentials, $U_{b}$ and $U_{B}$. We first review the more familiar situation of a codimension- 1 brane in a $5 \mathrm{D}$ bulk, and then contrast this with the codimension- 2 case with 6 bulk dimensions. (The situation for higher codimension is sketched in Appendix C.) Because they are peripheral to our main point we neglect gravitational effects in what follows, and so assume the mass scales involved are low enough for this to represent a good approximation.

\subsection{Codimension-one}

We first consider the codimension-one case, reproducing the results of ref. [10]. Consider the following 5D scalar field theory, having both bulk- and brane-localized interactions,

$$
S=-\int \mathrm{d}^{4} x \mathrm{~d} y\left[\frac{1}{2}\left(\partial_{M} \phi \partial^{M} \phi\right)+U_{B}(\phi)+\delta(y) U_{b}(\phi)\right]
$$

with $\left\{x^{M}\right\}=\left\{x^{\mu}, y\right\}$. The field equation for this model is

$$
\partial^{M} \partial_{M} \phi-U_{B}^{\prime}(\phi)=\delta(y) U_{b}^{\prime}(\phi)
$$

and the integration of this equation across the brane position (assuming continuity of $\phi$ ) further implies the scalar jump condition

$$
\left[\partial_{y} \phi\right]_{0}=U_{b}^{\prime}\left(\phi_{0}\right)
$$

where $\phi_{0}=\phi(y=0)$ and $[A]_{0}=A\left(y=0^{+}\right)-A\left(y=0^{-}\right)$. The classical energy density per unit brane volume associated with a given field configuration in this model is then

$$
\mathcal{H}=\int_{y_{\min }}^{y_{\max }} \mathrm{d} y\left[\frac{1}{2}\left(\dot{\phi}^{2}+(\nabla \phi)^{2}+\left(\partial_{y} \phi\right)^{2}\right)+U_{B}(\phi)\right]+U_{b}\left(\phi_{0}\right)
$$

where $y_{\min }<0<y_{\max }$ and $\nabla$ denotes differentiation in the in-brane spatial directions, $\left\{x^{i}\right\}$.

We now specialize to the case where the field has only a mass term in the bulk, while it has a quartic interaction on the brane. Keeping in mind that $\phi$ has dimension (mass) ${ }^{3 / 2}$ in 5 dimensions,

$$
U_{B}(\phi)=\frac{1}{2} m_{B}^{2} \phi^{2} \quad \text { and } \quad U_{b}(\phi)=-\frac{1}{2} m_{b} \phi^{2}+\frac{1}{4 M_{b}^{2}} \phi^{4},
$$

where $m_{b}>0$ is chosen to ensure that the minimum of the brane potential occurs at the nonzero value $\phi^{2}=M_{b}^{2} m_{b}$, in contrast with the bulk potential which is minimized at $\phi=0$.

Since $U_{b}$ and $U_{B}$ are not minimized by the same configuration, the vacuum solution need not correspond to a constant field configuration, $\partial_{M} \phi=0$. Since the solutions to the field equations that only depend on $y$ are exponentials, $\phi \propto e^{ \pm m_{B} y}$, the general bulk solution is a linear combination of such terms. If the extra dimension is sufficiently large $-\left|m_{B} y_{\text {min }}\right| \gg 1$ 
and $m_{B} y_{\max } \gg 1$ - then we can drop the solutions which grow exponentially far from the brane, just as if the extra dimension were noncompact. In this case the vacuum configuration should vanish at infinity, and the solution is therefore given by

$$
\phi(y)=\bar{\phi} e^{-m_{B}|y|},
$$

where $\bar{\phi}$ is to be fixed using the boundary condition, eq. (2.3), at $y=0$ : i.e. $-2 m_{B} \bar{\phi}=U_{b}^{\prime}(\bar{\phi})$, or

$$
\left(2 m_{B}-m_{b}+\frac{\bar{\phi}^{2}}{M_{b}^{2}}\right) \bar{\phi}=0
$$

When $m_{b}<2 m_{B}$ the only real solution allowed is $\bar{\phi}=0$, but when $m_{b}>2 m_{B}$ there are three solutions for $\bar{\phi}$, corresponding to $\bar{\phi}=0$ and $\bar{\phi}= \pm \phi_{c}$, with

$$
\phi_{c}^{2}=M_{b}^{2}\left(m_{b}-2 m_{B}\right) \text {. }
$$

Since $\mathcal{H}=0$ for $\bar{\phi}=0$ and $\mathcal{H}=-\frac{1}{4} M_{b}^{2}\left(m_{b}-2 m_{B}\right)^{2}$ for $\bar{\phi}^{2}=\phi_{c}^{2}$, we see that it is the nontrivial configuration which represents the classical ground state when $m_{b}>2 m_{B}$. This can also be seen more generally by writing the energy density as a function of $\bar{\phi}$,

$$
\mathcal{H}(\bar{\phi})=-\frac{1}{2}\left(m_{b}-2 m_{B}\right) \bar{\phi}^{2}+\frac{1}{4 M_{b}^{2}} \bar{\phi}^{4},
$$

which is indeed minimized, for $m_{b}>2 m_{B}$, by $\bar{\phi}= \pm M_{b} \sqrt{m_{b}-2 m_{B}}$, with the unstable stationary point, $\bar{\phi}=0$, situated at a local maximum.

The resulting vacuum

$$
\phi^{2}(y)=M_{b}^{2}\left(m_{b}-2 m_{B}\right) e^{-2 m_{B}|y|},
$$

extrapolates from the bulk minimum $(\phi=0)$ for large $y$ to the value $\phi_{0}= \pm M_{b} \sqrt{m_{b}-2 m_{B}}$ at the brane. This represents a compromise between the bulk minimum, the value $\bar{\phi}= \pm M_{b} \sqrt{m_{b}}$, which minimizes $U_{b}$, and the gradient energy required to interpolate between the two. Notice that $\phi_{0}$ approaches the brane minimum in the limit where the bulk potential is very flat, $m_{B} \ll m_{b}$.

\subsection{Codimension-two}

We now contrast the previous results with a similar analysis for the codimension-2 case of a real scalar field coupled to a 3-brane in 6 spacetime dimensions, where we show that the larger gradient energy more strongly favors the minimum of the bulk potential relative to that of the brane. Using the action

$$
S=-\int \mathrm{d}^{4} x \mathrm{~d}^{2} y\left[\frac{1}{2}\left(\partial_{M} \phi \partial^{M} \phi\right)+U_{B}(\phi)+\delta^{2}(y) U_{b}(\phi)\right],
$$

we have the equation of motion

$$
\partial^{M} \partial_{M} \phi-U_{B}^{\prime}(\phi)=\delta^{2}(y) U_{b}^{\prime}(\phi) .
$$


Assuming a flat space-time metric

$$
\mathrm{d} s^{2}=\eta_{\mu \nu} \mathrm{d} x^{\mu} \mathrm{d} x^{\nu}+\mathrm{d} r^{2}+r^{2} \mathrm{~d} \theta^{2},
$$

and integration of the equation of motion across a very small disc centered on the brane position at $r=0$ (assuming continuity of $\phi$ ) further implies the condition

$$
\lim _{r \rightarrow 0}\left[2 \pi r \partial_{r} \phi\right]=U_{b}^{\prime}\left(\phi_{0}\right)
$$

where $r$ measures the radial distance from the brane situated at $r=0$. For configurations depending only on $r$, this corresponds to using the radial field equation

$$
\frac{1}{r} \partial_{r}\left(r \partial_{r} \phi\right)-U_{B}^{\prime}(\phi)=\frac{\delta_{+}(r)}{2 \pi r} U_{b}^{\prime}(\phi),
$$

where $\delta_{+}(r)$ is normalized so that $\int_{0}^{a} \mathrm{~d} r \delta_{+}(r) f(r)=f(0)$, for any $a>0$.

Since our interest is in how the system resolves the frustration of minimizing brane and bulk potentials having different minima, we specialize to the simple choices

$$
U_{B}(\phi)=\frac{1}{2} m_{B}^{2} \phi^{2} \quad \text { and } \quad U_{b}(\phi)=-\frac{1}{2} \lambda_{2} \phi^{2}+\frac{1}{4} \lambda_{4} \phi^{4}
$$

with both $\lambda_{2}$ and $\lambda_{4}$ taken to be positive. Keeping in mind a $6 \mathrm{D}$ scalar field has dimension $(\text { mass })^{2}$, we see that the parameter $\lambda_{2}$ is dimensionless, while $\lambda_{4}=1 / M_{b}^{4}$.

Provided the extra dimensional radius, $L$, satisfies $m_{B} L \gg 1$, it is a good approximation to demand the bulk vacuum configuration to vanish at large $r$, leading to the following solution

$$
\phi(r)=\bar{\phi} K_{0}\left(m_{B} r\right)
$$

where the modified Bessel function, $K_{0}(z)$, falls exponentially with $z$ for large $z$ and diverges logarithmically as $z$ approaches zero. Using $K_{0}(z)=-\ln (z / 2)-\gamma+O(z)$ to evaluate $r \partial_{r} \phi \rightarrow$ $-\bar{\phi}$ as $r \rightarrow 0$, allows the boundary condition, eq. (2.14), to be written

$$
-2 \pi \bar{\phi}=U_{b}^{\prime}\left(\phi_{0}\right)
$$

and here we encounter the first difference from the codimension-2 case: $\phi(r)$ diverges logarithmically as $r \rightarrow 0$, making $\phi_{0}=\phi(r=0)$ ill defined. Regularizing ${ }^{1}$ by evaluating at a small but nonzero radius, $r=\epsilon$, gives $\phi_{\epsilon}=\bar{\phi} z_{\epsilon}$, where

$$
z_{\epsilon} \equiv K_{0}\left(m_{B} \epsilon\right)=\ell+\ln 2-\gamma+\mathcal{O}(\epsilon)
$$

with $\ell=-\ln \left(m_{B} \epsilon\right)$ diverging logarithmically when $\epsilon \rightarrow 0$ and $\gamma=0.5772 \ldots$ being the Euler-Mascheroni constant.

\footnotetext{
${ }^{1}$ This regularization can be done more precisely by modelling the codimension- 2 brane by a small codimension-1 circle at radius $r=\epsilon$, and using the codimension-1 jump conditions to relate the exterior bulk fields to the nonsingular fields in the circle's interior [11, 12].
} 
The trouble here lies in the fact that the classical solution for the bulk field coupled to a brane diverges when evaluated at the brane source. This is a completely generic feature for branes having codimension 3 or larger - e.g. the divergence of the Coulomb field at the position of the source charge. It is also generic for codimension 2, although exceptions in this case also arise, such as for the conical singularities arising in the static gravitational fields sourced by some [13, 14, 15] but not all [16, 11] codimension-2 branes. And the generic resolution to this problem lies in the need to renormalize the brane-bulk couplings even at the classical level [17, 18]. As these references show (and is briefly summarized in Appendix B), the requirement that bulk $\phi$ propagators be finite implies the brane couplings also diverge logarithmically in the limit $\epsilon \rightarrow 0$, with the result

$$
\lambda_{2}=\frac{\bar{\lambda}_{2}}{1+\bar{\lambda}_{2} \hat{\ell} / 2 \pi} \quad \text { and } \quad \lambda_{4}=\frac{\bar{\lambda}_{4}}{\left(1+\bar{\lambda}_{2} \hat{\ell} / 2 \pi\right)^{4}},
$$

where the $\bar{\lambda}_{i}$ are renormalized quantities that remain finite in the limit that $\epsilon \rightarrow 0$, and

$$
\hat{\ell}=-\ln (\mu \epsilon)=\ell+\ln \left(\frac{m_{B}}{\mu}\right)
$$

for an arbitrary renormalization scale $\mu$. For later purposes we remark that because the term in the action involving $\lambda_{2}$ is quadratic in $\phi$, it is possible to evaluate the classical scalar propagator, including the brane-bulk mixing, without having to assume that $\lambda_{2}$ or $\bar{\lambda}_{2}$ are small (see Appendix B for details). In particular the domain of validity of eq. (2.20) includes the regime of large $\bar{\lambda}_{2}$.

If we regularize by replacing $\phi_{0}$ with $\phi_{\epsilon}$, the boundary condition which determines $\bar{\phi}$ becomes

$$
-2 \pi r \partial_{r} \phi+U_{b}^{\prime}(\phi)=2 \pi \bar{\phi}+U_{b}^{\prime}\left(\phi_{\epsilon}\right)=\left(2 \pi-\lambda_{2} z_{\epsilon}+\lambda_{4} z_{\epsilon}^{3} \bar{\phi}^{2}\right) \bar{\phi}=0 .
$$

For $\lambda_{2}<2 \pi / z_{\epsilon}$ this only admits the trivial solution, $\bar{\phi}=0$, but for $\lambda_{2}>2 \pi / z_{\epsilon}$ three solutions are possible: $\bar{\phi}=0$ and $\bar{\phi}= \pm \phi_{c}$, with

$$
\phi_{c}^{2}=\frac{\left(\lambda_{2}-2 \pi / z_{\epsilon}\right)}{\lambda_{4} z_{\epsilon}^{2}} .
$$

Notice that the criterion distinguishing the existence of one or three solutions depends only logarithmically on $m_{B}$ (through its appearance in $z_{\epsilon}$ ), and can be phrased in a regularizationindependent manner by trading $\lambda_{2}$ for $\bar{\lambda}_{2}$. In particular, the condition $\lambda_{2}<2 \pi / z_{\epsilon}$ ensuring only $\bar{\phi}=0$ is a solution then becomes $\bar{\lambda}_{2}<2 \pi / c$, where $c=\ln 2-\gamma-\ln \left(m_{B} / \mu\right)$ defines the finite part of $z_{\epsilon} \equiv \hat{\ell}+c$.

The physical content of these expressions becomes clearer once the relative energy of these solutions is computed using the classical energy density, $\mathcal{H}(\bar{\phi})$, which is finite once it is expressed in terms of the renormalized quantities $\bar{\lambda}_{i}$. Explicitly, we have

$$
\mathcal{H}=\lim _{\epsilon \rightarrow 0}\left\{2 \pi \int_{\epsilon}^{\infty} r \mathrm{~d} r\left[\frac{1}{2}\left(\partial_{r} \phi\right)^{2}+\frac{1}{2} m_{B}^{2} \phi^{2}\right]+U_{b}\left(\phi_{\epsilon}\right)\right\}
$$




$$
=\lim _{\epsilon \rightarrow 0}\left\{\pi \bar{\phi}^{2} \int_{m_{B} \epsilon}^{\infty} \mathrm{d} z z\left[\left(K_{0}^{\prime}\right)^{2}+\left(K_{0}\right)^{2}\right]+U_{b}\left(\phi_{\epsilon}\right)\right\}
$$

The integral may be evaluated in closed form (see Appendix A), to give

$$
\begin{aligned}
\mathcal{H} & =\lim _{\epsilon \rightarrow 0}\left\{-\frac{\pi}{2} \bar{\phi}^{2} m_{B}^{2} \epsilon^{2} K_{0}\left(m_{B} \epsilon\right)\left[K_{0}\left(m_{B} \epsilon\right)-K_{2}\left(m_{B} \epsilon\right)\right]-\frac{1}{2} \lambda_{2} \phi_{\epsilon}^{2}+\frac{1}{4} \lambda_{4} \phi_{\epsilon}^{4}\right\} \\
& =\lim _{\epsilon \rightarrow 0}\left\{\pi \bar{\phi}^{2} z_{\epsilon}-\frac{1}{2} \lambda_{2} \bar{\phi}^{2} z_{\epsilon}^{2}+\frac{1}{4} \lambda_{4} \bar{\phi}^{4} z_{\epsilon}^{4}+\mathcal{O}(\epsilon)\right\}
\end{aligned}
$$

which uses the asymptotic form $K_{2}\left(m_{B} \epsilon\right) \simeq 2 /\left(m_{B} \epsilon\right)^{2}$ for small $\epsilon$. Using the asymptotic limit of eq. (2.20) for $\bar{\lambda}_{2} \hat{\ell} \gg 2 \pi$,

$$
\lambda_{2} \simeq \frac{2 \pi}{\hat{\ell}}\left[1-\left(\frac{2 \pi}{\bar{\lambda}_{2} \hat{\ell}}\right)+\cdots\right] \quad \text { and } \quad \lambda_{4} \simeq\left(\frac{2 \pi}{\bar{\lambda}_{2} \hat{\ell}}\right)^{4} \bar{\lambda}_{4}+\cdots,
$$

we find the finite limit

$$
\mathcal{H}=\frac{1}{2} g_{2} \bar{\phi}^{2}+\frac{1}{4} g_{4} \bar{\phi}^{4} \quad \text { with } \quad g_{2}=2 \pi\left(\frac{2 \pi}{\bar{\lambda}_{2}}-c\right) \quad \text { and } \quad g_{4}=\left(\frac{2 \pi}{\bar{\lambda}_{2}}\right)^{4} \bar{\lambda}_{4},
$$

where $c=\ln 2-\gamma-\ln \left(m_{B} / \mu\right)$, as above.

Notice the kinetic energy has combined with the bulk potential energy to partially cancel the quadratic term in the brane potential, with the solution $\bar{\phi}=0$ being energetically preferred for $\bar{\lambda}_{2}<2 \pi / c$ - the same criterion found earlier. Notice also that $c>0$ if $\mu>\mu_{\star}=\frac{1}{2} e^{\gamma} m_{B} \simeq$ $0.89 m_{B}$, and $c<0$ if $\mu<\mu_{\star}$. $c$ vanishes at the dividing case, $\mu=\mu_{\star}$, at which point the quadratic term is simply

$$
g_{2}=\frac{4 \pi^{2}}{\lambda_{2 \star}}
$$

with $\lambda_{2 \star} \equiv \bar{\lambda}_{2}\left(\mu_{\star}\right)$. In terms of renormalized quantities the criterion for symmetry breaking becomes $\lambda_{2 \star}<0$, in which case the scalar v.e.v. is

$$
\phi_{c}^{2}=-\frac{g_{2}}{g_{4}}=-\frac{\lambda_{2 \star}^{3}}{4 \pi^{2} \bar{\lambda}_{4}} .
$$

These calculations illustrate how the vacuum energetics of a bulk scalar depends crucially on the codimension of the brane to which it is coupled. In all cases the competition between gradient and potential energies in general allows the brane potential to drag the bulk scalar v.e.v. away from the value which minimizes $U_{B}$. But in the codimension- 1 case the marginal strength of brane instability which distinguishes a nonzero from a vanishing v.e.v., $m_{b}=2 m_{B}$, depends strongly on the UV-sensitive scale $m_{B}$. By contrast, the corresponding criterion for codimension-2 branes, $\bar{\lambda}_{2}=2 \pi / c$, is comparatively insensitive to $m_{B}$ because it is the larger gradient energies which replace $U_{B}$ in dominating the fight against $U_{b}$. (The situation for higher codimension is explored in Appendix O, below.) 


\subsection{The Self-Localized State}

Since we expect the quadratic term in $\mathcal{H}$ to describe the mass of small fluctuations about the background configuration, there is a potential puzzle hidden in the weak dependence of $g_{2}$ on $m_{B}$. To see why, suppose the two extra dimensions are a square torus of volume $V_{2}=L^{2}$, for which in the absence of the brane interactions we would normally expect a Kaluza Klein spectrum to be labelled by two integers, $n_{1}$ and $n_{2}$, with masses

$$
M_{n_{1} n_{2}}^{2}=m_{B}^{2}+M_{c}^{2}\left(n_{1}^{2}+n_{2}^{2}\right) \geq m_{B}^{2}
$$

where $M_{c}=2 \pi / L$. The puzzle is that all of these states have masses larger than $m_{B}$, a result which seems hard to reconcile with a mass governed by the size of the quadratic term, $\frac{1}{2} g_{2} \bar{\phi}^{2}$, of $\mathcal{H}$.

We next show that the resolution of this puzzle lies in the existence of a lower-mass 'bound' state whose mass lies in the gap, $m<m_{B}$, and whose presence relies on the influence of the interactions between $\phi$ and the brane. Furthermore, this state is localized near the brane by these interactions, in the sense that its wave-function falls exponentially away from the brane, with a characteristic size of order $a_{B} \sim 1 / k$, where $k^{2}=m_{B}^{2}-m^{2}$. We call this the self-localized state, inasmuch as its localization is a direct consequence of the scalarbrane interactions (rather than due to a geometric effect, like warping, such as considered in ref. [19]).

\section{The Fluctuation Spectrum}

To this end consider small fluctuations in the bulk scalar field,

$$
\phi(t, r, \theta)=\varphi(r)+\Phi_{n \omega}(r) e^{i n \theta-i \omega t},
$$

labelled by their energy, $\omega$, and angular momentum, ${ }^{2} n . \varphi(r)$ here denotes any of the vacuum configurations described above. The field equation obtained by linearizing eq. (2.12) in polar coordinates is

$$
\frac{1}{r} \partial_{r}\left(r \partial_{r} \Phi_{n \omega}\right)-\frac{n^{2}}{r^{2}} \Phi_{n \omega}-k^{2} \Phi_{n \omega}=\frac{\delta_{+}(r)}{2 \pi r}\left(-\lambda_{2}+3 \lambda_{4} \varphi^{2}\right) \Phi_{n \omega}
$$

where $k^{2}=m_{B}^{2}-\omega^{2}$. For the purposes of identifying the bound state we further specialize to axially symmetric modes, and so set $n=0$.

The steps for solving for $\Phi_{\omega}$ closely parallel those taken above to find the background solution. Away from $r=0$ the bulk solution is a linear combination of the modified Bessel functions, $K_{0}(k r)$ and $I_{0}(k r)$, although in the limit $k L \gg 1$ the admixture of $I_{0}(k r)$ can be made negligibly small. In this case the background configuration is $\varphi=\bar{\phi} K_{0}\left(m_{B} r\right)$ and the

\footnotetext{
${ }^{2}$ We assume here an axially-symmetric bulk, such as might be generated (say) by two branes.
} 
fluctuation solutions are well approximated by ${ }^{3}$

$$
\Phi_{\omega}(r)=N_{\omega} K_{0}(k r)
$$

with $N_{\omega}$ an appropriate normalization constant (e.g. $N_{\omega}^{2}=k^{2} / \pi$ when $k L \gg 1$ ). (In this notation the tower of KK states having masses greater than $m_{B}$ correspond to the ordinary Bessel functions obtained when $k$ is pure imaginary.) The eigenvalue, $k$, is obtained by imposing the boundary condition at $r=0$, which becomes

$$
2 \pi N_{\omega}+U_{b}^{\prime \prime}(\varphi) \Phi_{\omega}(r=0)=\left(2 \pi-\lambda_{2} \hat{z}_{\epsilon}+3 \lambda_{4} z_{\epsilon}^{2} \hat{z}_{\epsilon} \bar{\phi}^{2}\right) N_{\omega}=0
$$

where $z_{\epsilon}=-\ln \left(m_{B} \epsilon / 2\right)-\gamma=\hat{\ell}+c$ is as defined above, and $\hat{z}_{\epsilon}$ is the same quantity with $m_{B} \rightarrow k$ : i.e. $\hat{z}_{\epsilon}=z_{\epsilon}+\ln \left(m_{B} / k\right)$. This equation is to be read as being solved for $k$, leading to the result $\hat{z}_{\epsilon}=2 \pi /\left(\lambda_{2}-3 \lambda_{4} z_{\epsilon}^{2} \bar{\phi}^{2}\right)$, or

$$
\begin{aligned}
\ln \left(\frac{k}{m_{B}}\right) & =z_{\epsilon}-\frac{2 \pi}{\lambda_{2}-3 \lambda_{4} z_{\epsilon}^{2} \bar{\phi}^{2}}=\hat{\ell}+c-\frac{2 \pi}{\lambda_{2}-3 \lambda_{4}(\hat{\ell}+c)^{2} \bar{\phi}^{2}} \\
& =c-\left(2 \pi / \bar{\lambda}_{2}\right)-\left(3 \bar{\lambda}_{4} \bar{\phi}^{2} / \bar{\lambda}_{2}\right)\left(2 \pi / \bar{\lambda}_{2}\right)^{3}+\mathcal{O}(1 / \hat{\ell}) \\
& \rightarrow-\left(\frac{2 \pi}{\lambda_{2 \star}}\right)\left[1+\left(\frac{12 \pi^{2} \bar{\lambda}_{4} \bar{\phi}^{2}}{\lambda_{2 \star}^{3}}\right)\right] \quad \text { as } \epsilon \rightarrow 0 .
\end{aligned}
$$

Consequently, $k=m_{B} e^{-2 \pi / \lambda_{2 \mathrm{eff}}}$, or

$$
\omega^{2}=m_{B}^{2}-k^{2}=m_{B}^{2}\left[1-e^{-4 \pi / \lambda_{2 \mathrm{eff}}}\right]
$$

where

$$
\frac{1}{\lambda_{2 \mathrm{eff}}}=\frac{1}{\lambda_{2 \star}}\left[1+\left(\frac{12 \pi^{2} \bar{\lambda}_{4} \bar{\phi}^{2}}{\lambda_{2 \star}^{3}}\right)\right]
$$

Clearly this state lives in the gap, with $\omega<m_{B}$, provided only that $\lambda_{2 \text { eff }}>0$, and this mass can be hierarchically small if $\lambda_{2 \text { eff }} \gg 4 \pi$ (which lies within the domain of validity of the approximations used, as emphasized in Appendix B).

There are now two cases to consider. When $\lambda_{2 \star}>0$ we have $\bar{\phi}=0$ and so $\lambda_{2 \text { eff }}=\lambda_{2 \star}>0$, showing that the self-localized state exists. In the limit $\lambda_{2 \star} \gg 4 \pi$ we find $k \simeq m_{B}$ and $\omega^{2} \simeq 4 \pi m_{B}^{2} / \lambda_{2 \star}=g_{2} m_{B}^{2} / \pi \simeq g_{2} N_{\omega}^{2}$, in agreement with the result computed from $\mathrm{d}^{2} \mathcal{H} / \mathrm{d} \bar{\phi}^{2}$ (once care is taken to canonically normalize the $4 \mathrm{D}$ scalar field). Alternatively, when $\lambda_{2 \star}<0$ we have $\bar{\phi}= \pm \phi_{c}$, with $\phi_{c}$ given by eq. 2.30), and so $\lambda_{2 \mathrm{eff}}=-\frac{1}{2} \lambda_{2 \star}>0$. Again a bound state exists whose mass agrees with the result, $-2 g_{2} N_{\omega}^{2}$, obtained by differentiating $\mathcal{H}(\bar{\phi})$.

\footnotetext{
${ }^{3}$ Intriguingly, recasting the field equation to remove the single-derivative term, through the redefinition $\phi=\psi / r^{1 / 2}$, leads to the Schrödinger equation for motion of a point particle in a $1 / r^{2}$ potential supplemented by a $\delta$-function at the origin. This much-studied equation is known to exhibit the interesting phenomena of dimensional transmutation 20] and nontrivial limit cycles 21.
} 


\section{A Self-Localized Bulk Higgs and the Hierarchy Problem}

Because the above vacuum energetics show that the expectation value of a bulk scalar coupled to a codimension-2 (or higher codimension) brane is less sensitive to the details of the model's ultraviolet completion it can be used to provide a new approach to tackling the stability issue of the hierarchy problem. This section builds a simple illustrative example of this mechanism, in order to get a sense of its implications.

\subsection{The Model}

The mechanism's defining assumption is that the usual Standard Model Higgs doublet, $H(x, y)$, is located in an extra-dimensional bulk, while all of the other Standard Model particles - i.e. its gauge fields, $A_{\mu}^{a}(x)$, and fermions, $\psi_{k}(x)$ - reside on a brane whose codimension is at least two. (In practice we focus on the codimension-2 case in what follows, but generalizations to more general codimension are conceptually straightforward.) We take the brane potential to prefer an $S U_{L}(2) \times U_{Y}(1)$ breaking phase, while the bulk potential favors $S U_{L}(2) \times U_{Y}(1)$ invariance:

$$
U_{B}=m_{B}^{2} H^{*} H \quad \text { and } \quad U_{b}=-\lambda_{2} H^{*} H+\lambda_{4}\left(H^{*} H\right)^{2},
$$

where $m_{B}^{2}, \lambda_{2}$ and $\lambda_{4}$ are all real and positive (evaluated at $m_{B} \epsilon \ll 1$ ).

We have seen that the classical vacuum of the higher-dimensional theory depends crucially on the sign of the renormalized coupling, $\lambda_{2 \star}$, defined at the (large) scale $\mu_{\star} \simeq 0.89 \mathrm{~m}_{B}$. Notice in this regard that eq. 2.20) implies that both signs of $\lambda_{2 \star}$ can be consistent with positive $\lambda_{2}$ when $\ell=-\ln \left(m_{B} \epsilon\right)$ is sufficiently large. We take $\lambda_{2 \star}<0$ in order to ensure that the total classical energy is minimized by an $S U_{L}(2) \times U_{Y}(1)$ breaking configuration.

If we had had $S U_{L}(2) \times U_{Y}(1)$ invariance throughout the bulk we would at this point be able to perform a gauge transformation to ensure that the Higgs doublet everywhere

takes the unitary gauge form, $H=\frac{1}{\sqrt{2}}(0, \chi)^{T}$, with $\chi$ real. However because we only have gauge invariance at the brane position this choice can only be made at $y^{m}=0: H_{0}=$ $\frac{1}{\sqrt{2}}\left(0, \chi_{0}\right)^{T}$, where $H_{0} \equiv H(x, 0)$. Away from the brane $H$ in general contains 4 real fields, $H=\frac{1}{\sqrt{2}}\left(\zeta_{1}+i \zeta_{2}, \chi+i \zeta_{3}\right)^{T}$, each of which must solve its appropriate field equations.

The arguments of the previous sections imply that the classical vacuum solutions may be constructed in terms of $K_{0}\left(m_{B} r\right)$ and $I_{0}\left(m_{B} r\right)$, with the coefficient of $I_{0}\left(m_{B} r\right)$ negligibly small when the extra dimensions are large compared with $m_{B}^{-1}-$ i.e. $m_{B} L \gg 1$ :

$$
\zeta_{i}(r)=\bar{\zeta}_{i} K_{0}\left(m_{B} r\right) \quad \text { and } \quad \chi(r)=\bar{\chi} K_{0}\left(m_{B} r\right) .
$$

As before the normalizations, $\bar{\zeta}_{i}$ and $\bar{\chi}$, are determined by the boundary conditions at $r=0$, and so $\bar{\zeta}_{i}=0$ due to the choice of unitary gauge at the brane, which implies $\zeta_{i}(0)=0$ there. By contrast, the arguments of previous sections go through verbatim to imply $\bar{\chi} \equiv V^{2}$, with

$$
V^{4}=-\frac{g_{2}}{g_{4}}=-\frac{\lambda_{2 \star}^{3}}{(2 \pi)^{2} \bar{\lambda}_{4}}=-\frac{\lambda_{2 \star}^{3}}{(2 \pi)^{3}} M_{b}^{4},
$$


where we define $\bar{\lambda}_{4} / 2 \pi=1 / M_{b}^{4}$.

Similar arguments for the fluctuations, $\delta H$, show that in general all four components, $\delta \zeta_{i}$ and $\delta \chi$, are nonzero in the bulk. However the choice of unitary gauge at the brane endows $\delta \zeta_{i}$ with the boundary condition that it must vanish, and this in turn implies that none of these fields localizes at the branes in the same way that $\delta \chi$ does.

Since $S U(2) \times U(1)$ is only a global symmetry in the bulk, one might worry that its breaking by $H$ implies that the $\delta \zeta_{i}$ contain KK towers of Goldstone modes that are systematically light compared with $m_{B}$. These could be phenomenologically dangerous, even if their couplings must be derivatively suppressed [22]. However (as shown in appendix D in more detail) the only Goldstone modes in the bulk-Higgs sector are the three self-localized states for the fields $\delta \zeta_{i}$ that are eaten by the brane gauge fields via the usual Higgs mechanism. All other states with energies smaller than $m_{B}$ are typically removed by the boundary condition that requires $\delta \zeta_{i}$ to vanish at the brane, leaving the lightest remaining bona fide KK modes in $\delta \zeta_{i}$ with a mass of order $m_{B}$.

\subsection{Scales and Naturalness}

We now ask how $V$ depends on the other scales in the problem, in order to identify whether the choices required to have sufficiently small masses for electroweak gauge bosons are technically natural - i.e. stable against integrating out very heavy degrees of freedom.

The model potentially involves several scales: among which are the compactification scale, $M_{c}$; the scale of extra-dimensional gravity, $M_{*} \gg M_{c}$, (or perhaps the string scale), which controls our neglect of gravitational physics; the scale of brane structure, ${ }^{4} \Lambda=1 / \epsilon$, used in earlier regularizations, and so on. In principle the UV scale, $M \gg M_{c}$, to which we imagine being potentially sensitive, can be any one of these, or some other scale associated with other types of heavy particles.

Our choices of scales are restricted by the domain of validity of approximations used in our calculations. For instance, use of codimension- 2 branes without resolving the brane structure when discussing the UV physics implies $\Lambda \gg M$. Ignoring (for simplicity) the influence of the second brane on the mode functions (i.e. dropping the admixture of $I_{0}(k r)$ ) assumes $k \gg M_{c}$, where $k^{2}=m_{B}^{2}-m^{2}$ for the self-localized mode. Neglect (for convenience) of gravitational effects requires both $M_{*} \gg M_{c}$, and the condition that the spacetime curvatures generated by the configurations of interest to be small compared with $M_{*}^{2}$. For instance if $H$ takes values of order $V^{2}$ that change over distances of order $\epsilon$, then the resulting gradient energies do not overly gravitate if $(\partial H)^{2} / M_{*}^{6} \sim\left(V / M_{*}\right)^{4}\left(\Lambda / M_{*}\right)^{2} \ll 1$. In what follows we assume all of these conditions to hold. The question we ask is not whether these hierarchies themselves are stable under renormalization (as this would require more information, such as specifying a stabilization mechanism for the size of the extra dimensions), but rather whether the choices required of the Higgs potential to obtain an acceptably small $V$ are stable against renormalization, given the presence of these (and possibly other) scales.

\footnotetext{
${ }^{4}$ For instance, such structure might ultimately arise if the 3-brane were really a higher-dimensional brane wrapped about further, smaller extra dimensions.
} 
This requires an estimate of the corrections to $U_{B}$ and $U_{b}$ that might arise as various kinds of heavy particles are integrated out. Although a precise statement of this requires specifying the theory's UV completion, some generic statements are possible on dimensional grounds for the corrections due to integrating out heavy particles that interact through small dimensionless couplings. This is because if such a particle has a large mass $M$, then its generic contribution to a coupling, $\lambda_{i}$, having dimension (mass) ${ }^{n}$ is $\delta \lambda_{i} \propto M^{n}$. According to this kind of estimate we expect

$$
\delta m_{B}^{2} \propto M^{2}, \quad \delta \lambda_{2} \propto \ln M \quad \text { and } \quad \delta \lambda_{4} \propto M^{-4}
$$

As a result it is natural to expect the corrections to $m_{B}$ to be dominated by the heaviest particles that can contribute, and so generically expect $m_{B}$ to be comparable to the largest scales in the problem (and in particular to satisfy $m_{B} \gg M_{c}$ and $m_{B} \gg M_{W}$ ). It is the large size of these contributions to $m_{B}$ that underlie the usual formulation of the hierarchy problem in 4 dimensions, because in this case the scale of the Higgs v.e.v. turns out to be proportional to $\left|m_{B}\right|$.

By contrast, in the $6 \mathrm{D}$ model of present interest we have seen that the size of the Higgs v.e.v. is largely independent of $m_{B}$, depending dominantly on the dimensionless coupling $\bar{\lambda}_{2}$ and the dimensionful coupling $\bar{\lambda}_{4}$. But $\bar{\lambda}_{2}$ is dimensionless, and so tends to depend only logarithmically on the large UV scale $M$. Potentially more dangerous is $\bar{\lambda}_{4} / 2 \pi=1 / M_{b}^{4}$ since this more directly sets the size of $V$. However this is also not UV sensitive because corrections to it vary inversely with the relevant particle mass on dimensional grounds, and so are dominated by the contributions of the lightest particles, rather than the heaviest.

As stated above, we emphasize that our goal here is not to provide an ultraviolet completion of the bulk-Higgs model, as would be required to understand in detail the conditions necessary to produce a large hierarchy in the first place, as this goes beyond the scope of this paper. Our goal is instead to point out how the introduction of Higgs bulk-brane couplings allows interestingly different mass-dependence in low-energy observables, and to study what this might imply for the low-energy sector.

\subsection{Higgs-induced Mass Terms}

The phenomenology of any such Higgs hinges on the form of its couplings to observed Standard Model particles, which are assumed in this framework to be localized on a brane.

\section{Gauge Couplings}

At first sight it is bizarre to restrict the SM gauge fields to a brane and yet allow a charged matter field (the Higgs doublet) live in the bulk. This is bizarre because the $S U_{L}(2) \times U_{Y}(1)$ symmetry transformations are global transformations in the bulk (since there is no spinone field there to 'gauge' them), yet are local on the brane. Nonetheless, it must be possible because we could imagine the UV completion of the brane of interest being an ordinary gauge-

Higgs theory containing vortex- or domain-wall-type defects. Since the Higgs field defining 
the defect typically vanishes at the interior of such a vortex/domain-wall, there generically should be spin-1 states which would be very massive given the nonzero Higgs in the bulk, but which can remain light by being localized on the brane. ( $D$-branes also contain localized spin-1 fields.)

More precisely, it can be shown that gauge invariance of such a theory can always be ensured through an appropriate choice of effective interactions (or counter-terms) on the brane. Slightly generalizing the discussion of ref. 223] to codimension two, we may see this formally by taking the Higgs covariant derivatives to be

$$
D_{M} H(x, y)=\partial_{M} H(x, y)-\delta^{2}(y) \delta_{M}^{\mu} i \kappa g A_{\mu}^{a}(x) T_{a} H(x, y) .
$$

Here $T_{a}$ are gauge generators, and as before the $x^{\mu}$ lie along the brane directions while the $y^{m}$ are transverse. $g$ here denotes the dimensionless gauge coupling on the brane and $\kappa$ is a dimensionful constant, required in order to counter the dimensions of the delta function. One reason for the need for brane counter-terms can be seen because the off-brane components of this covariant derivative are not actually covariant under the gauge transformation

$$
\delta H(x, y)=\delta^{2}(y) i \kappa \Omega^{a}(x) T_{a} H(x, y)
$$

even when supplemented by the standard $x^{\mu}$-dependent nonabelian transformations of $A_{\mu}^{a}(x)$. They are not because there is no gauge potential in $D_{m} H=\partial_{m} H$ to cancel the term arising when the derivative acts on the delta function. There is however a counterterm that can be added on the brane such that the entire combination is gauge invariant.

The implications of a bulk Higgs v.e.v. for gauge boson masses can be seen by writing out the bulk and brane kinetic terms

$$
\begin{gathered}
\mathcal{L}_{\text {kin }}=-\int \mathrm{d}^{2} y D_{M} H^{*} D^{M} H-\kappa_{b} \mathcal{D}_{\mu} H_{0}^{*} \mathcal{D}^{\mu} H_{0} \\
=-\int \mathrm{d}^{2} y\left[\partial_{M} H^{*} \partial^{M} H\right]-\left(\kappa+\kappa_{b}\right) \mathcal{D}_{\mu} H_{0}^{*} \mathcal{D}^{\mu} H_{0}+\kappa \partial_{\mu} H_{0}^{*} \partial^{\mu} H_{0} \\
+\frac{\kappa g^{2}}{2}\left[1-\kappa \delta^{2}(0)\right]\left(H_{0}^{*}\left\{T_{a}, T_{b}\right\} H_{0}\right) A_{\mu}^{a} A^{b \mu},
\end{gathered}
$$

where $\mathcal{D}_{\mu} H_{0} \equiv \partial_{\mu} H_{0}-i g A_{\mu}^{a} T_{a} H_{0}$ is the standard covariant derivative on the brane. This shows that all of the gauge-boson mass terms appear in the brane kinetic term provided $\kappa \delta^{2}(0)=1$ (and so $\kappa=O\left(\epsilon^{2}\right)$ ). Notice that this implies $\kappa+\kappa_{b} \sim \kappa_{b}$ for any scale $\kappa_{b} \gg O\left(\epsilon^{2}\right)$.

Superficially the gauge-boson mass obtained from these equations diverges as $\epsilon \rightarrow 0$, due to the divergence there of $H_{0}$. However, this divergence is countered by the renormalization of all Higgs-brane interactions due to the generic 'dressing' of these couplings [17, 18] by the Higgs-brane mixing, $\lambda_{2}$ :

$$
\kappa_{b}=\frac{\bar{\kappa}_{b}}{\left(1+\bar{\lambda}_{2} \hat{\ell} / 2 \pi\right)^{2}} .
$$

Going to unitary gauge at the brane position, for which $H_{0}=\frac{1}{\sqrt{2}}\left(0, \chi_{0}\right)^{T}$, with $\langle\chi\rangle=$ $V^{2} K_{0}\left(m_{B} r\right)$, we then have $\kappa_{b}\left\langle\chi_{0}\right\rangle^{2}=\bar{\kappa}_{b} V^{4}\left(2 \pi / \lambda_{2 \star}\right)^{2}$ as $\epsilon \rightarrow 0$. 
The $S U_{L}(2) \times U_{Y}(1)$ doublet structure of the Higgs then leads in the standard way to the prediction $M_{Z}=M_{W} / \cos \theta_{W}$, where $\theta_{W}$ is the weak mixing angle, and the $W$-boson mass is, $M_{W}=\frac{1}{2} g v$, with

$$
v^{2}=\left(\frac{2 \pi}{\lambda_{2 \star}}\right)^{2} \bar{\kappa}_{b} V^{4}=(246 \mathrm{GeV})^{2} .
$$

Taking $\bar{\kappa}_{b}=1 / f^{2}$, this shows that successful phenomenology requires $V^{2}=f v\left(\left|\lambda_{2 \star}\right| / 2 \pi\right)$ : i.e. $V$ is the geometric mean between $246 \mathrm{GeV}$ and the scale $\left|\lambda_{2 \star}\right| f / 2 \pi$ :

$$
V \sim 10^{9} \mathrm{GeV}\left(\frac{\left|\lambda_{2 \star}\right| f / 2 \pi}{10^{15} \mathrm{GeV}}\right)^{1 / 2} .
$$

Recall that within the present framework we have $V^{4}=\left|\lambda_{2 \star} / 2 \pi\right|^{3}\left(2 \pi / \bar{\lambda}_{4}\right)-$ c.f. eq. (3.3) — so defining $M_{b}$ by $\bar{\lambda}_{4} / 2 \pi=1 / M_{b}^{4}$ as before we see that eq. (3.9) also implies that $M_{b}$ must be of order

$$
M_{b} \sim \sqrt{v f}\left(\frac{2 \pi}{\left|\lambda_{2 \star}\right|}\right)^{1 / 4} .
$$

This requires either $M_{b} \sim f \sqrt{2 \pi /\left|\lambda_{2 \star}\right|} \sim v$, or a hierarchy $v \ll M_{b} \ll f \sqrt{2 \pi /\left|\lambda_{2 \star}\right|}$, if $f \sqrt{2 \pi /\left|\lambda_{2 \star}\right|} \gg v$. In the absence of a symmetry which forbids a Higgs kinetic term but allows a quartic $\left(H^{*} H\right)^{2}$ interaction on the brane, naturalness argues we should take $f$ and $M_{b}$ to be the same order of magnitude, in which case any hierarchy between $M_{b}$ and $v$ must be due to $\left|\lambda_{2 \star}\right| / 2 \pi$ being very large or very small. Furthermore, having $f$ and $M_{b}$ both larger than $v$ requires $\left|\lambda_{2 \star}\right| / 2 \pi \lesssim O(1)$.

\section{Fermion Couplings}

Fermion masses in this picture are similarly given by Yukawa couplings between brane-based fermions, $\psi_{k}$, and the bulk Higgs doublet. In unitary gauge on the brane, $H_{0}=\frac{1}{\sqrt{2}}\left(0, \chi_{0}\right)^{T}$, these have the form

$$
\mathcal{L}_{\text {yuk }}=\frac{y_{i j}}{F}\left(\bar{\psi}_{i} \psi_{j}\right) \chi_{0},
$$

for $y_{i j}$ a collection of dimensionless Yukawa couplings, and $F$ representing an appropriate ultraviolet scale. The resulting fermion masses are

$$
m_{i j}=\frac{y_{i j}}{F}\left\langle\chi_{0}\right\rangle=\frac{2 \pi \bar{y}_{i j} V^{2}}{\lambda_{2 \star} F}
$$

with

$$
y_{i j}=\frac{\bar{y}_{i j}}{1+\bar{\lambda}_{2} \hat{\ell} /(2 \pi)}
$$

being the renormalized Yukawa coupling, as required to counter the divergence of $H$ at the brane position, and the second equality in eq. (3.13) uses $y_{i j}\left\langle\chi_{0}\right\rangle=2 \pi \bar{y}_{i j} V^{2} / \lambda_{2 \star}$ in the limit $\epsilon \rightarrow 0$, where $\langle\chi\rangle=V^{2} K_{0}\left(m_{B} r\right)$. 
Since $\mathcal{L}_{\text {yuk }}$ breaks flavor symmetries - unlike the Higgs kinetic terms - the scale $F$ need not be of the same order of magnitude $\mathrm{as}^{5} f$. In particular, since the dominant contributions to couplings having dimensions of inverse mass come from the lightest scales to contribute, $F$ is typically set by the smallest UV scale which involves flavor-violating physics while $f$ can be much smaller than this. Because of this eqs. (3.9) and (3.13) may contain the seeds of an explanation of the observed smallness of most fermion masses relative to those of the electroweak gauge bosons, since

$$
\frac{m_{i j}}{M_{W}}=\frac{\bar{y}_{i j}}{g}\left(\frac{2 f}{F}\right) .
$$

Even a mild hierarchy, $F \gg f$, removes some of the burden of having to require $\bar{y}_{i j} / g$ to be very small.

\subsection{Couplings to the Higgs Fluctuations}

We have seen that the spectrum of fluctuations in the Higgs field generically contains an assortment of KK modes, many of whose masses start above a large gap, $m_{K K} \gtrsim m_{B}$. For $m_{B}$ sufficiently large these modes need not play an important role in low-energy observables. The two exceptions to the above statement are the bulk Goldstone modes, whose masses are generically of order $M_{c}$, and the self-localized state whose mass can lie within the gap below $m_{B}$, and be hierarchically smaller if $\left|\lambda_{2 \star}\right| \gg 2 \pi$. Furthermore, this latter state is present regardless of whether or not the Higgs v.e.v. is nonzero. These light states are likely to be the ones relevant to Higgs phenomenology in Bulk Higgs models, and so this section computes their couplings.

\section{The bulk Goldstone modes}

The simplest couplings to compute are those of the bulk Goldstone modes, $\delta \zeta_{i}$, because their vanishing at the brane position guarantees they completely drop out of any brane couplings that depend only on $H_{0}$ or $\partial_{\mu} H_{0}$, and not on off-brane derivatives like $\partial_{m} H_{0}$. In particular this ensures their removal (in unitary gauge) from the fermion Yukawa couplings and gauge couplings described above.

\section{The self-localized state}

Normalizing the wave-function of the self-localized state in the extra dimensions gives a canonically normalized $4 \mathrm{D}$ state $h$, where $\chi=h(x) N_{\omega} K_{0}(k r)$, so $y_{i j} \chi=\left(2 \pi / \lambda_{2 \star}\right)(k / \sqrt{\pi}) \bar{y}_{i j} h$, with $k^{2}=m_{B}^{2}-m_{h}^{2}$. The couplings of $h$ to fermions are then given by interactions of the form

$$
\mathcal{L}_{4 D}=\frac{2 \pi \bar{y}_{i j}}{\lambda_{2 \star}}\left(\frac{k}{\sqrt{\pi} F}\right)\left(\bar{\psi}_{i} \psi_{j}\right) h
$$

\footnotetext{
${ }^{5}$ This could arise, say, if the 3 -brane is really a higher-dimensional brane wrapped in extra dimensions, and the flavor structure is associated with this wrapping, since this would suggest $F \simeq \Lambda \gg f$.
} 
leading to dimensionless 'physical' Yukawa couplings of order

$$
\hat{y}_{i j}=\frac{2 \pi \bar{y}_{i j}}{\lambda_{2 \star}}\left(\frac{k}{\sqrt{\pi} F}\right)=y_{i j}^{\mathrm{sm}}\left(\frac{m_{B}}{\sqrt{\pi} f}\right)\left(\frac{2 \pi}{\lambda_{2 \star}}\right) e^{-4 \pi /\left|\lambda_{2 \star}\right|}
$$

where the argument of the exponential assumes $\lambda_{2 \star}<0$ (as required for a nonzero Higgs v.e.v.), and the last equality compares to what would be expected in the SM:

$$
y_{i j}^{\mathrm{sm}} \equiv \frac{m_{i j}}{v}=\bar{y}_{i j}\left(\frac{f}{F}\right) .
$$

Notice that the quantity $\left(2 \pi /\left|\lambda_{2 \star}\right|\right) \exp \left[-4 \pi /\left|\lambda_{2 \star}\right|\right]$ falls to zero for large and small $\left|\lambda_{2 \star}\right|$, taking the maximum value of 0.18 when $\left|\lambda_{2 \star}\right| / 2 \pi=2$.

These expressions show that the self-localized Higgs couplings, $\hat{y}_{i j}$, can differ significantly

from what would be expected in the SM, for two reasons. First, $\hat{y}_{i j}$ can be larger than $y_{i j}^{\mathrm{sm}}$ if $m_{B} \gg f$, and if sufficiently large the self-localized state becomes a strongly coupled broad resonance. Second, $\hat{y}_{i j}$ can differ from $y_{i j}^{\mathrm{sm}}$ because of its dependence on $\lambda_{2 \star} / 2 \pi$, which acts to suppress $\hat{y}_{i j} / y_{i j}^{\mathrm{sm}}$ in the limit that $\left|\lambda_{2 \star}\right| / 2 \pi$ is either very large or very small. This possibility of having $\hat{y}_{i j}$ differ from the SM expectation contrasts with $4 \mathrm{D}$ intuition based on the couplings of a single scalar whose v.e.v. generates mass, since such a scalar must have couplings given by the ratio $m_{i j} / v$. The reason this conclusion does not hold in the extra-dimensional case is that because the v.e.v., $\langle H(x, 0)\rangle$, responsible for generating masses generically receives contributions from many KK modes and not just the v.e.v. of the single $4 \mathrm{D}$ self-localized state, $h$.

\section{Possible Signatures of a Bulk Higgs Scenario}

We next sketch some of the qualitative signatures and constraints that might be expected for the kind of Higgs scenario described above. What is to be expected depends somewhat on the choices made for the various scales in the problem, so we divide the discussion according to four simple options according to whether or not we take $\left|\lambda_{2 \star}\right|$ to be large or small, and whether we take $M_{c} \sim 1 \mathrm{TeV}$, or $M_{c} \sim 10^{-2} \mathrm{eV}$ (as for large-extra-dimensional models).

\subsection{Inclusive Processes}

We first consider inclusive processes for which a specific Higgs state is not measured, and so which involve a summation over all possible KK modes. These are largely insensitive to the specifics of individual modes, such as the details of the self-localized state.

\section{Fermion-fermion scattering}

An important inclusive observable is the rate for fermion-fermion scattering mediated by a virtual Higgs. The amplitude for this process is of order

$$
\mathcal{A}\left(\psi_{i} \psi_{j} \rightarrow H \rightarrow \psi_{r} \psi_{s}\right) \simeq \frac{y_{i j} y_{r s}}{F^{2}} i G_{p}(0 ; 0) \delta^{4}\left(p_{i}+p_{j}-p_{r}-p_{s}\right),
$$


where $p^{\mu} \equiv\left(p_{i}+p_{j}\right)^{\mu}=\left(p_{r}+p_{s}\right)^{\mu}$. Here $G_{p}\left(y ; y^{\prime}\right)$ is the bulk Higgs propagator, Fourier transformed in the brane directions, $x^{\mu}$, but evaluated in position space in the off-brane directions, $y^{m} . G_{p}(0 ; 0)$ denotes the same quantity evaluated at the brane position, and is given (see Appendix $\mathrm{B}$ for details) in terms of the corresponding propagator in the absence of brane-Higgs couplings, $D_{p}\left(y ; y^{\prime}\right)$, by

$$
G_{p}(0 ; 0)=\frac{D_{p}(0 ; 0)}{1-i \lambda_{2} D_{p}(0 ; 0)}
$$

Eliminating $y_{i j}, y_{r s}$ and $\lambda_{2}$ in terms of the renormalized quantities, $\bar{y}_{i j}, \bar{y}_{r s}$ and $\bar{\lambda}_{2}$, and taking $\epsilon \rightarrow 0$, we find the finite result

$$
\begin{aligned}
\mathcal{A}\left(\psi_{i} \psi_{j} \rightarrow H \rightarrow \psi_{r} \psi_{s}\right) & \simeq \frac{\bar{y}_{i j} \bar{y}_{r s}}{\bar{\lambda}_{2} F^{2}}\left[\frac{1}{1-i \bar{\lambda}_{2} D_{p}^{\mu}(0 ; 0)}\right] \delta^{4}\left(p_{i}+p_{j}-p_{r}-p_{s}\right) \\
& \simeq \frac{y_{i j}^{\mathrm{sm}} y_{r s}^{\mathrm{sm}}}{\bar{\lambda}_{2} f^{2}}\left[\frac{1}{1-i \bar{\lambda}_{2} D_{p}^{\mu}(0 ; 0)}\right] \delta^{4}\left(p_{i}+p_{j}-p_{r}-p_{s}\right),
\end{aligned}
$$

where $i D_{p}^{\mu}(0 ; 0)=(1 / 2 \pi) \ln (\mu / P)$, where $P^{2}=p^{2}+m_{B}^{2}$.

If this same process were computed using the exchange of a massive 4D SM Higgs scalar, we'd have instead obtained

$$
\mathcal{A}^{\mathrm{sm}}\left(\psi_{i} \psi_{j} \rightarrow H \rightarrow \psi_{r} \psi_{s}\right) \simeq y_{i j}^{\mathrm{sm}} y_{r s}^{\mathrm{sm}}\left[\frac{1}{p^{2}+m_{H}^{2}}\right] \delta^{4}\left(p_{i}+p_{j}-p_{r}-p_{s}\right)
$$

and so the leading effect is to replace the scale $p^{2}+m_{H}^{2}$ by $\bar{\lambda}_{2} f^{2}\left[1-i \bar{\lambda}_{2} D_{p}^{\mu}(0 ; 0)\right]$. The absence of an observed signal therefore implies the order-of-magnitude bound

$$
\bar{\lambda}_{2} f^{2}\left[1+\frac{\bar{\lambda}_{2}}{2 \pi} \ln \left(\frac{P}{\mu}\right)\right] \gtrsim O(100 \mathrm{GeV})^{2},
$$

where $P^{2}=\left(p_{i}+p_{j}\right)^{2}+m_{B}^{2}=\left(p_{r}+p_{s}\right)^{2}+m_{B}^{2}$.

If reactions of this type were to mediate flavor-changing neutral currents, the strong restrictions on these could potentially bound the scale $F$ to be quite large. However, because the Yukawa couplings can have the same flavor structure as in the SM, there can be a GIM mechanism at work [24] that naturally suppresses the dangerous flavor-changing neutral current (FCNC) reactions produced by bulk-Higgs exchange. We henceforth assume this to be true, and therefore do not further worry about bounds on the fermion couplings due to FCNCs.

\section{Vacuum Polarization}

As is well known, the contributions to loops of the SM Higgs is well constrained by precision electroweak measurements. The main source of these contributions is through the Higgs contribution to the vacuum polarization of the electroweak gauge bosons. For an extradimensional bulk Higgs, this contribution is of order

$$
\Pi_{a b}^{\mu \nu}(p) \simeq g^{2} \kappa_{b}^{2} \operatorname{tr}\left(T_{a} T_{b}\right) \int \frac{\mathrm{d}^{4} q}{(2 \pi)^{4}}(2 p-q)^{\mu}(2 p-q)^{\nu} i G_{q}(0 ; 0) i G_{p-q}(0 ; 0)
$$




$$
\simeq \frac{g^{2} \bar{\kappa}_{b}^{2}}{\bar{\lambda}_{2}^{2}} \operatorname{tr}\left(T_{a} T_{b}\right) \int \frac{\mathrm{d}^{4} q}{(2 \pi)^{4}}\left[\frac{(2 p-q)^{\mu}(2 p-q)^{\nu}}{\left[1-i \bar{\lambda}_{2} D_{p}^{\mu}(0 ; 0)\right]\left[1-i \bar{\lambda}_{2} D_{p-q}^{\mu}(0 ; 0)\right]}\right]
$$

plus a possible tadpole term. Since the remaining integration, $\mathrm{d}^{4} q$, diverges in the ultraviolet it must be regularized, and this is most conveniently done using dimensional regularization.

Of most interest for phenomenological purposes is the contribution to the oblique parameters $S, T$ and $U$ [1, 25], which involve those terms in $\Pi_{a b}^{\mu \nu}$ having the tensor structure $\left(p^{2} \eta^{\mu \nu}-p^{\mu} p^{\nu}\right)$. Since the Higgs is an $S U_{L}(2) \times U_{Y}(1)$ doublet, it automatically preserves the accidental custodial $S U_{c}(2)$ symmetry [1, 26] that preserves the successful mass relation $M_{W}=M_{Z} \cos \theta_{W}$, thereby suppressing its contribution to $T$ and making $S$ of most interest. Because all mass dependence in eq. (4.6) is logarithmic, recalling the definition $\bar{\kappa}_{b}=1 / f^{2}$ and extracting the conventional factors of $g^{2} / 4 \pi$, we obtain the estimate

$$
S \sim \frac{1}{4 \pi \bar{\lambda}_{2}^{2}}\left(\frac{p^{4}}{f^{4}}\right)
$$

where $p^{2}$ represents the momentum transfer of interest. Applied to LEP experiments we may take $p^{4}=M_{Z}^{4}$ and $|S|<0.1$ to conclude $\bar{\lambda}_{2} f^{2} \gtrsim v^{2}$.

\subsection{Higgs Decays to Fermions}

Another class of observables involve specifying a specific Higgs KK mode. Perhaps the simplest of these is the decay rate for specific Higgs states into SM particles (although this decay need not dominate the lifetime of a given KK mode because it must also compete with other channels, such as off-brane decays into the Goldstone modes $\delta \zeta_{i}$ ).

\section{Generic KK states}

For simplicity we start with the decay of a generic KK mode into brane fermions, assuming the KK wave-functions, $\Psi(y)$, extend throughout much of the extra-dimensional bulk so that $|\Psi(0)|^{2} \simeq 1 / V_{2} \simeq M_{c}^{2}$. Once excited, such a heavy state can decay through the interaction (3.12), with the rate

$$
\Gamma\left(\chi \rightarrow \bar{\psi}_{i} \psi_{j}\right) \simeq|\Psi(0)|^{2} \frac{\left|y_{i j}\right|^{2}}{F^{2}} M_{\chi} \simeq\left|y_{i j}\right|^{2}\left(\frac{M_{c}}{F}\right)^{2} M_{\chi}
$$

where $M_{\chi} \geq m_{B}$ is the mass of the decaying mode. (Recall that the bulk Goldstone modes, $\delta \zeta_{i}$, do not decay in this way because of the requirement that they vanish at the brane.) We see that $\Gamma \ll M_{\chi}$ naturally follows from the smallness of the quantities $y_{i j}$ and $M_{c} / F$ (the

latter of which is particularly small in the case of large extra dimensions). Whether these are the dominant decay channels depends on the availability of light states in the bulk (or on other branes) into which competing decays can proceed, and how efficiently these Higgs decays occur. 


\section{The self-localized state}

Notice that $y_{i j}$ vanishes, strictly speaking, when $\epsilon \rightarrow 0$ with $\bar{y}_{i j}$ and $\bar{\lambda}_{2}$ held fixed (making eq. (4.8) vanish logarithmically in this limit). The same is not true of the self-localized state, whose wave-function also diverges logarithmically at the position of the brane as $\epsilon \rightarrow 0$. In this case the decay rate can be computed using the interaction of eq. (3.16), leading (on neglect of final-state fermion masses) to the standard 4D expression

$$
\Gamma\left(h \rightarrow \bar{\psi}_{i} \psi_{j}\right)=\frac{1}{8 \pi}\left|\hat{y}_{i j}\right|^{2} m_{h}=\Gamma^{\mathrm{sm}}\left(h \rightarrow \bar{\psi}_{i} \psi_{j}\right)\left(\frac{m_{B}^{2}}{\pi f^{2}}\right)\left(\frac{2 \pi}{\lambda_{2 \star}}\right)^{2} e^{-16 \pi /\left|\lambda_{2 \star}\right|},
$$

which remains nonzero as $\epsilon \rightarrow 0$. This drops dramatically, as required in the unlocalized limit, as $\left|\lambda_{2 \star}\right| \rightarrow 0$, and scales as $\Gamma^{\mathrm{sm}} m_{h}^{4} /\left(16 \pi m_{B}^{2} f^{2}\right)$ when $\left|\lambda_{2 \star}\right| \gg 2 \pi$.

\subsection{TeV-Scale Compactifications}

Suppose, first, the compactification scale, $M_{c}$, lies in the $\mathrm{TeV}$ range and so, in the absence of significant warping, the $4 \mathrm{D}$ Planck scale, $M_{p} \sim M_{*}^{2} / M_{c}$, comes out right if $M_{*} \sim 10^{10} \mathrm{GeV}$. This leaves lots of room to choose the other scales of interest to be much smaller than $M_{*}$ in order to justify our neglect of gravitational interactions. We do not speculate as to how the extra-dimensional size is stabilized at this scale.

Choosing $M_{c}$ this large also ensures that this is the mass of the lightest KK mode of the bulk Goldstone bosons, $\delta \zeta_{i}$, ensuring that these modes do not play much of a phenomenological role until energies are reached - at the LHC, God willing - that allow the direct production of KK excitations. The same is true of the generic KK modes of the field $\chi$, provided we also choose $m_{B}$ to be large enough.

We have seen that the absence of Higgs detection in oblique or in 2-fermion to 2-fermion

processes implies us to choose $f \sqrt{\left|\lambda_{2 \star}\right|}$ to be at least several hundred $\mathrm{GeV}$, whereas our use of a $6 \mathrm{D}$ calculational framework requires both $f$ and $M_{b} \sim \sqrt{v f}\left(2 \pi /\left|\lambda_{2 \star}\right|\right)^{1 / 4}$ to be $\gtrsim M_{c}$. There are then two subcategories to consider, depending on the size of $\left|\lambda_{2 \star}\right| / 2 \pi$.

\section{Weak localization}

Consider first the limit of small $\left|\lambda_{2 \star}\right|$, for which $m_{h} \rightarrow m_{B}$ and $k \rightarrow 0$. Because $k$ is small, the 'bound' state is not strongly localized relative to generic extra dimensional scales, and the breakdown of the approximation $k \gg M_{c}$ demands we go beyond the simple large-volume limits used above for the scalar v.e.v. and wave-function. Taking $\left|\lambda_{2 \star}\right| \sim 0.01$ for illustrative purposes, we see that requiring $f>M_{c} \sim$ few TeV automatically ensures $f \sqrt{\left|\lambda_{2 \star}\right|} \gtrsim$ several hundred $\mathrm{GeV}$, and so is large enough to avoid the phenomenological bounds.

For weak localization, the exponential suppression of $\hat{y}_{i j}$ for small $\left|\lambda_{2 \star}\right|$ allows us to choose $m_{B}$ to be much larger than $f$ without the Higgs-fermion couplings becoming strong. However we cannot have all $\chi$ states be too much higher than the $\mathrm{TeV}$ scale without there being a breakdown of the low-energy effective theory, such as through the development of unitarity problems in the scattering of longitudinal $W$ bosons that the SM would suffer in the absence 
of a low-energy Higgs particle [1, 27, 28], and this puts an upper bound on how large $m_{B}$ can be. In this case the $\chi$ spectrum resembles the usual intuition for bulk fields in the absence of brane couplings, consisting of a tower of Higgs KK modes starting above the gap at $m_{B}$. Furthermore, because these particles are likely to have a significant decay rate into the lighter bulk Goldstone states, any observed Higgs is likely to have a significant invisible width.

Because $m_{B}$ cannot be made exceedingly large without running into troubles, and because $M_{c}$ is typically smaller, it should be possible to observe some of the Higgs KK states at the LHC. Although the mass- $M_{c}$ Goldstone states cost less energy, they are more difficult to produce because of the absence of direct couplings to the initial brane-based SM particles. The most likely channel for doing so is the virtual excitation of KK modes of the bulk state $\chi$. Convincing evidence for these Goldstone states together with an absence for KK modes for the electroweak gauge bosons would provide the smoking gun for this scenario: with the Higgs in the bulk but gauge interactions localized to live only on the branes.

\section{Strong localization}

In the opposite limit, $\left|\lambda_{2 \star}\right| \gg 2 \pi$, the lowest energy state becomes localized to the brane with $k \simeq m_{B}$, and its mass drops to $m_{h}^{2} \simeq 8 \pi m_{B}^{2} /\left|\lambda_{2 \star}\right| \ll m_{B}^{2}$. In this case $m_{B}$ can be higher than it could for weak localization, provided that the self-localized state is lighter than a few $\mathrm{TeV}$ and so can unitarize the scattering of longitudinal gauge boson modes.

An upper limit to how large $m_{B}$ can be is found from the condition that this light, localized Higgs state be weakly coupled

$$
\left|\frac{\hat{y}_{i j}}{y_{i j}^{\mathrm{sm}}}\right|^{2} \simeq \frac{8}{\pi}\left(\frac{m_{B}}{f}\right)^{2}\left|\frac{2 \pi}{\lambda_{2 \star}}\right|^{3} \simeq \frac{1}{8 \pi}\left(\frac{m_{h}}{f}\right)^{2}\left(\frac{m_{h}}{m_{B}}\right)^{4} .
$$

Large $\left|\lambda_{2 \star}\right|$ also implies that the condition $f>M_{c}$ automatically ensures the validity of the phenomenological limits that require $f \sqrt{\left|\lambda_{2 \star}\right|}$ to be larger than several hundred $\mathrm{GeV}$, and makes the strongest constraint on $f$ the theoretical condition that $M_{b}$ be larger than $M_{c}$.

For instance for moderately large $\left|\lambda_{2 \star}\right| / 2 \pi \sim 10^{2}$, then keeping $m_{h}$ at the TeV scale requires $m_{B} \simeq 10 \mathrm{TeV}$, and taking $M_{b} \sim 1 \mathrm{TeV}$ then implies $f \sim 10 \mathrm{TeV}$. By contrast, if $m_{B}$ should be the largest scale considered so far, $m_{B} \sim M_{*} \sim 10^{10} \mathrm{GeV}$, then $\left|\lambda_{2 \star}\right| / 2 \pi \sim 10^{20}$, and so $M_{b} \sim 10^{-5} f>1 \mathrm{TeV}$ implies a strong hierarchy between $M_{b}$ and $f>10^{5} \mathrm{GeV}$ whose naturality would have to be explained. Notice that the physical couplings, $\hat{y}_{i j}$, are much smaller than for the SM given these scales.

In this case $m_{B}$ could easily be large enough to preclude the direct detection of a Higgs KK spectrum, even at the LHC, leaving the burden of Higgs physics carried by the single self-localized Higgs state. In principle this can be distinguished from a SM Higgs in several ways. First, it could well have a large invisible width, if the mass of the self-localized state is sufficiently large compared with the mass, $M_{c}$, of the bulk Goldstone modes. Second, it can be distinguished by identifying the difference in the strength of its couplings to fermions from those expected in the SM. 


\subsection{Large Extra Dimensions}

An alternative choice [2, 15, 29] would put the scale of extra-dimensional gravity at $M_{*} \sim 10$ $\mathrm{TeV}$, which then requires $M_{c} \sim 10^{-2} \mathrm{eV}$. As a result, the upper bound $m_{B}<M_{*}$ automatically keeps the generic Higgs KK modes light enough to potentially be seen at the LHC, yet absence of the detection of Higgs KK modes also implies $m_{B}$ cannot be much below the TeV scale.

An automatic consequence of having $M_{c}$ so small is to make the bulk Goldstone states, $\delta \zeta_{i}$, essentially massless. This ensures that they are always kinematically available as final states for $\chi$ decays, making a significant invisible width for this state inevitable. In fact, the very lightest KK Goldstone modes in this scenario are light enough to mediate forces between macroscopic bodies, with generically near-gravitational strength, making them potentially relevant to precision tests of Newton's inverse-square law for gravity. Their presence is nonetheless unlikely to have been already ruled out due to the absence of direct couplings to brane matter, and the derivative nature of their Goldstone interactions.

In this scenario the conditions $f, M_{b} \gtrsim M_{c}$ pose no significant constraint, with more information coming from the phenomenological conditions that $f \sqrt{\left|\lambda_{2 \star}\right|}$ be larger than a few hundred GeV. Notice that if we also require $f \lesssim M_{*}$ then we must have an upper bound $\left|\lambda_{2 \star}\right| \lesssim 10^{4}$, and so the self-localized state cannot be more than a few orders of magnitude lighter than $m_{B}$.

Because the KK tower of modes is so narrowly spaced - by $O\left(M_{c}\right)$ - they provide almost a continuum of states. Although each of these modes couples with gravitational strength, their phase space makes their inclusive production cross section of order the weak-interaction size [2]. Once the Higgs is produced, its phenomenology is likely to resemble that of extradimensional gravitons [30] or other bulk matter fields [31], including likely large invisible decay channels.

\section{Conclusions}

In this paper we examine a new way for brane-world scenarios to change how we think about low-energy naturalness problems. We do so by showing how oft-neglected couplings to branes can dramatically change the vacuum energetics and low-energy spectrum for bulk scalar fields. In particular, we show that when coupled to codimension- 2 branes bulk scalar fields can have two unusual properties:

- They can acquire v.e.v.s that are only logarithmically related to the size of the UVsensitive quadratic term, $\frac{1}{2} m_{B}^{2} \phi^{2}$, in the bulk Higgs potential;

- They can acquire low-energy KK modes that are localized to the branes (without the need for warping), and whose mass can lie inside the naive gap below the energy set by the mass scale $m_{B}$.

We further use these two observations to explore the possibility of building phenomenological brane-world models for which all Standard Model particles (save the Higgs) are trapped 
on a brane, but with the Higgs allowed to live in the bulk. We estimate the size of the effective couplings of such a Higgs to gauge bosons and fermions on the brane, and use these to estimate the sizes of masses and couplings to the Higgs KK modes.

We do not try to identify ultraviolet completions of the bulk-Higgs model, and so do not identify at a microscopic level why the electroweak hierarchy exists in the first place. Our focus is instead on whether such a hierarchy can be technically natural purely within the low-energy theory. We identify in eq. (3.3) the main obstacle to systematically raising the UV scale of this effective theory above the weak scale, since this equation generically requires the two dimensionful parameters $f$ and $M_{b}$ - governing the size of the brane potential term $\left(H^{*} H\right)^{2} / M_{b}^{4}$ and the brane kinetic term $\left(D_{M} H^{*} D^{M} H\right) / f^{2}$ - either to satisfy $M_{b} \sim$ $f \sqrt{2 \pi / \lambda_{2 \star}}$ with both near the electroweak scale, or to satisfy the hierarchy $M_{b} \ll f \sqrt{2 \pi / \lambda_{2 \star}}$ if both are large compared with the electroweak scale. This latter hierarchy shows how the problem gets recast with a bulk Higgs, since both interactions are allowed by the same symmetries, making it unnatural for them to have coefficients suppressed by very different scales.

We provide a very preliminary discussion of possible signals and constraints on these models, including the observation that most realizations predict a significant invisible width for any observed 'Higgs', once detected. Simple estimates are made of Higgs decay rates into SM particles, the scattering rate for fermions due to virtual Higgs exchange, and the contribution of virtual Higgs loops to gauge boson vacuum polarization. These are used to outline the qualitative features of Higgs phenomenology within this class of models. In all cases we find that the phenomenology of these models is sufficiently interesting to bear further, more detailed study.

\section{Acknowledgements}

We wish to thank Georges Azuelos, Massimo Giovannini, Quim Matias and James Wells for helpful comments and suggestions. This research has been supported in part by funds from the Natural Sciences and Engineering Research Council (NSERC) of Canada. CB also acknowledges support from CERN, the Killam Foundation, and McMaster University. CdR is funded by an Ontario Ministry of Research and Information (MRI) postdoctoral fellowship. Research at the Perimeter Institute is supported in part by the Government of Canada through NSERC and by the Province of Ontario through MRI.

\section{References}

[1] For a recent summary of these issues see, C.P. Burgess and G.D. Moore, The Standard Model: A Primer, Cambridge University Press 2007.

[2] N. Arkani-Hamed, S. Dimopoulos and G. Dvali, Phys. Lett. B429 (1998) 263 (hep-ph/9803315); Phys. Rev. D59 (1999) 086004 (hep-ph/9807344); I. Antoniadis, N. Arkani-Hamed, S. Dimopoulos and G. R. Dvali, Phys. Lett. B 436, 257 (1998) [arXiv:hep-ph/9804398]. 
[3] L. Randall, R. Sundrum, Phys. Rev. Lett. 83 (1999) 3370 [hep-ph/9905221], Phys. Rev. Lett. 83 (1999) 4690 [hep-th/9906064].

[4] A. Pomarol and M. Quiros, Phys. Lett. B 438 (1998) 255 [arXiv:hep-ph/9806263];

A. Delgado, A. Pomarol and M. Quiros, Phys. Rev. D 60 (1999) 095008 [hep-ph/9812489];

I. Antoniadis, Phys. Lett. B 246, 377 (1990);

T. Gherghetta and A. Pomarol, Nucl. Phys. B 586 (2000) 141 [arXiv:hep-ph/0003129].

[5] L. J. Hall, Y. Nomura and D. R. Smith, Nucl. Phys. B 639 (2002) 307 [hep-ph/0107331];

I. Gogoladze, Y. Mimura and S. Nandi, Phys. Lett. B 562 (2003) 307 [hep-ph/0302176].

[6] G. Burdman and Y. Nomura, Nucl. Phys. B 656 (2003) 3 [hep-ph/0210257];

N. Haba and Y. Shimizu, Phys. Rev. D 67 (2003) 095001 [Erratum-ibid. D 69 (2004) 059902] [hep-ph/0212166];

N. Haba and T. Yamashita, JHEP 0404 (2004) 016 [hep-ph/0402157];

G. Martinelli, M. Salvatori, C. A. Scrucca and L. Silvestrini, JHEP 0510 (2005) 037

[hep-ph/0503179];

M. Sakamoto and K. Takenaga, Phys. Rev. D 75 (2007) 045015 [hep-th/0609067].

[7] C. A. Scrucca, M. Serone, L. Silvestrini and A. Wulzer, JHEP 0402 (2004) 049

[hep-th/0312267];

C. Biggio and M. Quiros, Nucl. Phys. B 703 (2004) 199 [hep-ph/0407348];

Y. Hosotani, S. Noda and K. Takenaga, Phys. Lett. B 607 (2005) 276 [hep-ph/0410193];

D. Hernandez, S. Rigolin and M. Salvatori, arXiv:0712.1980 [hep-ph].

[8] E. Dudas, C. Papineau and V. A. Rubakov, JHEP 0603, 085 (2006) [arXiv:hep-th/0512276].

[9] E. Dudas and C. Papineau, JHEP 0611, 010 (2006) [arXiv:hep-th/0608054].

[10] F. Coradeschi, S. De Curtis, D. Dominici and J. R. Pelaez, [arXiv:0712.0537 [hep-th]].

[11] J. Vinet and J. M. Cline, Phys. Rev. D 70 (2004) 083514 [hep-th/0406141].

[12] M. Peloso, L. Sorbo and G. Tasinato, Phys. Rev. D 73 (2006) 104025 [hep-th/0603026];

E. Papantonopoulos, A. Papazoglou and V. Zamarias, JHEP 0703 (2007) 002 [hep-th/0611311];

B. Himmetoglu and M. Peloso, Nucl. Phys. B 773 (2007) 84 [hep-th/0612140];

N. Kaloper and D. Kiley, JHEP 0705 (2007) 045 [hep-th/0703190];

C. P. Burgess, D. Hoover and G. Tasinato, JHEP 0709 (2007) 124 [arXiv:0705.3212 [hep-th]];

M. Minamitsuji and D. Langlois, Phys. Rev. D 76 (2007) 084031 [arXiv:0707.1426 [hep-th]];

F. Arroja, T. Kobayashi, K. Koyama and T. Shiromizu, JCAP 0712 (2007) 006 [arXiv:0710.2539 [hep-th]];

C. Bogdanos, A. Kehagias and K. Tamvakis, Phys. Lett. B 656 (2007) 112 [arXiv:0709.0873 [hep-th]].

[13] A. Vilenkin, Phys. Rev. D23 (1981) 852;

R. Gregory and C. Santos, Phys. Rev. D 56, 1194 (1997) [gr-qc/9701014].

[14] S. M. Carroll and M. M. Guica, [hep-th/0302067];

[15] Y. Aghababaie, C. P. Burgess, S. L. Parameswaran and F. Quevedo, Nucl. Phys. B 680 (2004) 389 [hep-th/0304256]. 
[16] G. W. Gibbons, R. Guven and C. N. Pope, Phys. Lett. B 595 (2004) 498 [hep-th/0307238];

Y. Aghababaie et al., JHEP 0309 (2003) 037 [hep-th/0308064];

P. Bostock, R. Gregory, I. Navarro and J. Santiago, Phys. Rev. Lett. 92 (2004) 221601

[hep-th/0311074];

C. P. Burgess, F. Quevedo, G. Tasinato and I. Zavala, JHEP 0411 (2004) 069 [hep-th/0408109];

J. Vinet and J. M. Cline, Phys. Rev. D 71 (2005) 064011 [hep-th/0501098].

[17] W. D. Goldberger and M. B. Wise, Phys. Rev. D 65 (2002) 025011 [arXiv:hep-th/0104170].

[18] C. de Rham, JHEP 0801 (2008) 060 [arXiv:0707.0884 [hep-th]];

C. de Rham, AIP Conf. Proc. 957, 309 (2007) [arXiv:0710.4598 [hep-th]].

[19] D. Langlois and M. Sasaki, Phys. Rev. D 68 (2003) 064012 [hep-th/0302069].

[20] W. Frank, D. J. Land and R. M. Spector, Rev. Mod. Phys. 43 (1971) 36;

G. Parisi and F. Zirilli, J. Math. Phys. 14 (1973) 243;

H. E. Camblong, L. N. Epele, H. Fanchiotti and C. A. Garcia Canal, Phys. Rev. Lett. 85 (2000) 1590 [hep-th/0003014];

S. R. Beane, P. F. Bedaque, L. Childress, A. Kryjevski, J. McGuire and U. v. Kolck, Phys. Rev. A 64 (2001) 042103 [quant-ph/0010073].

[21] E. Braaten and D. Phillips, [hep-th/0403168].

[22] See for instance, C. P. Burgess, Phys. Rept. 330 (2000) 193 [arXiv:hep-th/9808176].

[23] For a discussion of these issues in the codimension-1 case, see A. Kehagias and K. Tamvakis, Phys. Lett. B 628 (2005) 262 [hep-th/0507130].

[24] S. L. Glashow, J. Iliopoulos and L. Maiani, Phys. Rev. D 2 (1970) 1285.

[25] M. E. Peskin and T. Takeuchi, Phys. Rev. Lett. 65 (1990) 964; Phys. Rev. D 46 (1992) 381;

G. Altarelli and R. Barbieri, Phys. Lett. B 253 (1991) 161;

G. Altarelli, R. Barbieri and S. Jadach, Nucl. Phys. B 369 (1992) 3 [Erratum-ibid. B 376 (1992) 444];

C. P. Burgess, S. Godfrey, H. Konig, D. London and I. Maksymyk, Phys. Lett. B 326, 276

(1994) [hep-ph/9307337]; Phys. Rev. D 49, 6115 (1994) [hep-ph/9312291].

[26] S. Weinberg, Phys. Rev. D19, 1277 (1979);

L. Susskind, Phys. Rev. D20, 2619 (1979);

P. Sikivie, L. Susskind, M. Voloshin and V. Sakharov, Nucl. Phys. B173 (1980) 189;

H. Georgi and D. B. Kaplan, Phys. Lett. B 145 (1984) 216.

[27] J.M. Cornwall, D.N. Levin and G. Tiktopoulos, Phys. Rev. D10 1145 (1974);

B.W. Lee, C. Quigg and H. Thacker, Phys. Rev. D16 1519 (1977);

M. Veltman, Acta. Phys. Pol. B8 475 (1977).

[28] C. P. Burgess and D. London, Phys. Rev. D 48 (1993) 4337 [hep-ph/9203216]; Phys. Rev. Lett. 69 (1992) 3428.

[29] C. P. Burgess, J. Matias and F. Quevedo, Nucl. Phys. B 706 (2005) 71 [hep-ph/0404135].

[30] G. F. Giudice, R. Rattazzi and J. D. Wells, Nucl. Phys. B 544 (1999) 3 [hep-ph/9811291];

T. Han, J. D. Lykken and R. J. Zhang, Phys. Rev. D 59 (1999) 105006 [hep-ph/9811350]. 
[31] D. Atwood, C. P. Burgess, E. Filotas, F. Leblond, D. London and I. Maksymyk, Phys. Rev. D 63 (2001) 025007 [hep-ph/0007178];

I. Antoniadis and K. Benakli, Int. J. Mod. Phys. A 15 (2000) 4237 [hep-ph/0007226];

J. L. Hewett and D. Sadri, Phys. Rev. D 69 (2004) 015001 [hep-ph/0204063];

P. H. Beauchemin, G. Azuelos and C. P. Burgess, J. Phys. G 30 (2004) N17 [hep-ph/0407196];

J. Phys. G 31 (2005) 1 [hep-ph/0401125].

\section{A. Some Properties of Bessel Functions}

This appendix summarizes a few properties of modified Bessel functions which are used in the main text. The modified Bessel functions are linearly independent solutions to the differential equation

$$
z^{2} y^{\prime \prime}+z y^{\prime}-\left(z^{2}+\nu^{2}\right) y=0
$$

with $I_{\nu}(z)$ chosen to be regular at $z=0$ and $K_{\nu}(z)$ chosen to fall off to zero as $z \rightarrow \infty$. They are defined in terms of ordinary Bessel functions, $J_{\nu}(z)$, and Hankel functions, $H_{\nu}^{(1)}(z)$, by

$$
I_{\nu}(z)=i^{-\nu} J_{\nu}(i z) \quad \text { and } \quad K_{\nu}(z)=\frac{\pi}{2} i^{\nu+1} H_{\nu}^{(1)}(i z) .
$$

The expansion of these functions for small argument is used in the text. For $0<z \ll \sqrt{\nu+1}$ it is given by

$$
I_{\nu}(z) \simeq \frac{1}{\Gamma(\nu+1)}\left(\frac{z}{2}\right)^{\nu}, \quad K_{0}(z) \simeq-\ln \left(\frac{z}{2}\right)-\gamma \quad \text { and } \quad K_{\nu}(z) \simeq \frac{\Gamma(\nu)}{2}\left(\frac{2}{z}\right)^{\nu} \quad \text { if } \quad \nu>0
$$

The asymptotic form at large $z$ is similarly given (for $z \gg\left|\nu^{2}-\frac{1}{4}\right|$ ) by

$$
I_{\nu}(z) \simeq \frac{1}{\sqrt{2 \pi z}} e^{z} \quad \text { and } \quad K_{\nu}(z) \simeq \sqrt{\frac{\pi}{2 z}} e^{-z}
$$

The energy integral encountered in the main text can be evaluated explicitly, using the following Bessel-function identities

$$
K_{\nu}^{\prime}=-K_{\nu-1}-\frac{\nu K_{\nu}}{z}=-K_{\nu+1}+\frac{\nu K_{\nu}}{z}
$$

which imply in particular $K_{0}^{\prime}=-K_{1}, K_{1}^{\prime}=-K_{0}-K_{1} / z=-K_{2}+K_{1} / z$ and $K_{2}^{\prime}=-K_{1}-$ $2 K_{2} / z$. Repeated application of these shows that

$$
\frac{\mathrm{d}}{\mathrm{d} z}\left[\frac{1}{2} z^{2}\left(K_{0}^{2}-K_{1}^{2}\right)\right]=z K_{0}^{2} \quad \text { and } \quad \frac{\mathrm{d}}{\mathrm{d} z}\left[\frac{1}{2} z^{2}\left(K_{1}^{2}-K_{0} K_{2}\right)\right]=z K_{1}^{2},
$$

and so

$$
z\left(K_{0}^{2}+K_{1}^{2}\right)=\frac{\mathrm{d}}{\mathrm{d} z}\left[\frac{1}{2} z^{2} K_{0}\left(K_{0}-K_{2}\right)\right] .
$$




\section{B. Classical Divergences in Brane Couplings}

This appendix summarizes the derivation of the renormalization of the codimension- 2 couplings encountered in the text, with an emphasis on identifying its domain of validity.

Consider to this end the following bulk-brane quadratic action for a single real scalar field,

$$
S=-\frac{1}{2} \int \mathrm{d}^{4} x \mathrm{~d}^{2} y\left[\partial_{M} \phi \partial^{M} \phi+m_{B}^{2} \phi^{2}\right]+\frac{1}{2} \int d^{4} x \lambda_{2} \phi^{2} .
$$

(The unusual sign for the brane term is chosen to be consistent with its use in the main text.) The exact propagator, $G\left(x, y ; x^{\prime}, y^{\prime}\right)$, for this theory satisfies the differential equation

$$
\left[\partial_{M} \partial^{M}-m_{B}^{2}+\lambda_{2} \delta^{2}(y)\right] G\left(x, y ; x^{\prime}, y^{\prime}\right)=i \delta^{4}\left(x-x^{\prime}\right) \delta^{2}\left(y-y^{\prime}\right),
$$

while the propagator in the absence of the brane coupling, $D\left(x, y ; x^{\prime}, y^{\prime}\right)$, instead satisfies

$$
\left[\partial_{M} \partial^{M}-m_{B}^{2}\right] D\left(x, y ; x^{\prime}, y^{\prime}\right)=i \delta^{4}\left(x-x^{\prime}\right) \delta^{2}\left(y-y^{\prime}\right)
$$

It is useful to regard these as the position-basis representation of two abstract operators, $G$ and $D$, so that $G\left(x, y ; x^{\prime}, y^{\prime}\right)=\left\langle x, y|G| x^{\prime}, y^{\prime}\right\rangle$ (and similarly for $D$ ). In this case the above relations can be written $G^{-1}=D^{-1}-i V$, where $\left\langle x, y|V| x^{\prime}, y^{\prime}\right\rangle=\lambda_{2} \delta^{2}(y) \delta^{4}\left(x-x^{\prime}\right) \delta^{2}\left(y-y^{\prime}\right)$. Multiplying on the left by $D$ and on the right by $G$ then allows this to be written as $G=$ $D+i D V G$, whose position-basis expression is equivalent to the integral equation

$$
G\left(x, y ; x^{\prime}, y^{\prime}\right)=D\left(x, y ; x^{\prime}, y^{\prime}\right)+i \lambda_{2} \int \mathrm{d}^{4} \hat{x} D(x, y ; \hat{x}, 0) G\left(\hat{x}, 0 ; x^{\prime}, y^{\prime}\right) \text {. }
$$

After Fourier transforming the translation-invariant $x^{\mu}$ directions

$$
G\left(x, y ; x^{\prime}, y^{\prime}\right)=\int \frac{\mathrm{d}^{4} p}{(2 \pi)^{4}} G_{p}\left(y ; y^{\prime}\right) e^{i p \cdot\left(x-x^{\prime}\right)},
$$

eq. (B.4) becomes the exact statement

$$
G_{p}\left(y ; y^{\prime}\right)=D_{p}\left(y ; y^{\prime}\right)+i \lambda_{2} D_{p}(y ; 0) G_{p}\left(0 ; y^{\prime}\right)
$$

Since this no longer involves convolutions it may be solved explicitly. Specializing first to $y=0$ implies $G_{p}\left(0 ; y^{\prime}\right)=D_{p}\left(0 ; y^{\prime}\right) /\left[1-i \lambda_{2} D_{p}(0 ; 0)\right]$, which when re-substituted into eq. (B.6) gives

$$
G_{p}\left(y ; y^{\prime}\right)=D_{p}\left(y ; y^{\prime}\right)+i \lambda_{2} \frac{D_{p}(y ; 0) D_{p}\left(0 ; y^{\prime}\right)}{1-i \lambda_{2} D_{p}(0 ; 0)}
$$

Notice that no approximations have been made that implicitly restrict us to small $\lambda_{2}$.

The problem with the solution, eq. (B.7), is that the quantity $D_{p}(0 ; 0)$ diverges, and this observation lies at the root of the need for renormalization. The expression for $D_{p}\left(y ; y^{\prime}\right)$ may 
be explicitly constructed as the following mode sum, using polar coordinates $\left\{y^{m}\right\}=\{r, \theta\}$ in the transverse dimensions, with $r=0$ representing the brane position:

$$
D_{p}\left(r, \theta ; r^{\prime}, \theta^{\prime}\right)=-i \sum_{n=-\infty}^{\infty} e^{i n\left(\theta-\theta^{\prime}\right)} \int_{0}^{\infty}\left(\frac{q \mathrm{~d} q}{2 \pi}\right) \frac{1}{p^{2}+q^{2}+m_{B}^{2}} J_{n}(q r) J_{n}\left(q r^{\prime}\right)
$$

where ${ }^{6} p^{2}=p_{\mu} p^{\mu}$. To isolate the divergence in $D_{p}(0 ; 0)$ evaluate at $r=r^{\prime}=0$ and use $J_{n}(0)=\delta_{n 0}$ to get

$$
D_{p}^{\Lambda}(0 ; 0)=-i \int_{0}^{\Lambda}\left(\frac{q \mathrm{~d} q}{2 \pi}\right) \frac{1}{p^{2}+q^{2}+m_{B}^{2}}=-\frac{i}{2 \pi} \ln \left(\frac{\Lambda}{P}\right)+O\left(\frac{P^{2}}{\Lambda^{2}}\right),
$$

where $P^{2}=p^{2}+m_{B}^{2}$.

Renormalization may also be performed without resorting to an expansion in powers of $\lambda_{2}$. The goal is to redefine $\lambda_{2}=\bar{\lambda}_{2}(\Lambda) \rightarrow \bar{\lambda}_{2}(\mu)$ in such a way as to absorb the divergence in $D^{\Lambda}(0 ; 0)$ :

$$
\frac{\lambda_{2}}{1-i \lambda_{2} D_{p}^{\Lambda}(0 ; 0)} \equiv \frac{\bar{\lambda}_{2}(\mu)}{1-i \bar{\lambda}_{2}(\mu) D_{p}^{\mu}(0 ; 0)},
$$

or, equivalently

$$
\frac{1}{\bar{\lambda}_{2}(\Lambda)} \equiv \frac{1}{\bar{\lambda}_{2}(\mu)}+i\left[D_{p}^{\Lambda}(0 ; 0)-D_{p}^{\mu}(0 ; 0)\right]=\frac{1}{\bar{\lambda}_{2}(\mu)}+\frac{1}{2 \pi} \ln \left(\frac{\Lambda}{\mu}\right)
$$

in agreement with the usage in the main text.

\section{Higher codimension}

In this appendix we examine how the arguments of $\S 2$ change for a Higgs living in a $(4+n)$ dimensional bulk coupled to a codimension- $n$ brane, with $n \geq 3$.

We divide the discussion into a derivation of how the brane couplings renormalize in arbitrary codimension, and then examine the energy density that governs the size of the resulting scalar expectation value.

\section{C.1 Coupling renormalization}

We start with a discussion of brane coupling renormalization. The main complication in the higher-codimension case is the appearance of power-law divergences, with all of the pitfalls and complications which these entail for the low-energy description [28].

Consider the $(n+4)$-dimensional scalar field

$$
S=-\int \mathrm{d}^{4} x \mathrm{~d}^{n} y\left[\frac{1}{2}\left(\partial_{M} \phi \partial^{M} \phi\right)+\frac{1}{2} m_{B}^{2} \phi^{2}+\delta^{n}(y) V_{b}(\phi)\right],
$$

\footnotetext{
${ }^{6}$ The generalization of this expression to the case where the transverse geometry has a conical defect at the brane position is given in ref. 18].
} 
with brane potential

$$
V_{b}=-\frac{1}{2} \lambda_{2} \phi^{2}+\frac{1}{4} \lambda_{4} \phi^{4}
$$

living in a flat space-time with metric

$$
\mathrm{d} s^{2}=\eta_{\mu \nu} \mathrm{d} x^{\mu} \mathrm{d} x^{\nu}+\mathrm{d} r^{2}+r^{2} \gamma_{a b}(\theta) \mathrm{d} \theta^{a} \mathrm{~d} \theta^{b} .
$$

Here the $\theta^{a}$ are coordinates for the $n-1$ angular directions, whose total volume we denote by $\varpi=\int \mathrm{d}^{(n-1)} \theta \sqrt{\gamma}$. We focus for simplicity on spherically symmetric solutions (independent of the angular directions), although this assumption is not crucial (since higher modes in the angular directions are regular at $r=0$ ).

As for codimension 2, the relation between the propagator, $G$, in the presence of the brane coupling, and the propagator, $D$, in its absence, is

$$
G_{k}\left(y ; y^{\prime}\right)=D_{k}\left(y ; y^{\prime}\right)+i \lambda_{2} \frac{D_{k}(y ; 0) D_{k}\left(0 ; y^{\prime}\right)}{1-i \lambda_{2} D_{k}(0 ; 0)}
$$

and as before the need for renormalization may be traced to the divergence in $D_{k}(0 ; 0)$. The nature of this divergence can be divined from the mode sum giving the propagator, $D$, in the absence of brane couplings

$$
\left[\square-m_{B}^{2}\right] D\left(x, y ; x^{\prime}, y^{\prime}\right)=i \delta^{4}\left(x-x^{\prime}\right) \delta^{n}\left(y-y^{\prime}\right),
$$

which, in brane-Fourier space,

$$
D\left(x, y ; x^{\prime}, y^{\prime}\right)=\int \frac{\mathrm{d}^{4} p}{(2 \pi)^{4}} D_{p}\left(y ; y^{\prime}\right) e^{i p \cdot\left(x-x^{\prime}\right)},
$$

has as solution

$$
D_{p}\left(x ; x^{\prime}\right)=-i \int_{0}^{\infty} \frac{q^{n-1} \mathrm{~d} q}{\varpi} \frac{1}{p^{2}+m_{B}^{2}+q^{2}}\left[\frac{1}{(q r)^{\nu}} J_{\nu}(q r)\right]\left[\frac{1}{\left(q r^{\prime}\right)^{\nu}} J_{\nu}\left(q r^{\prime}\right)\right]+\cdots,
$$

with $\nu=(n-2) / 2$. The ellipses in this last equation represent those terms involving the nontrivial angular modes.

Using the asymptotic form for $J_{\nu}$ in the limit $q r \ll 1: J_{\nu}(q r)=(q r)^{\nu} /\left[\nu ! 2^{\nu}\right]+\mathcal{O}(q r)$, we find

$$
D_{p}\left(r=0 ; r^{\prime}=0\right)=-\frac{i}{(\nu !)^{2} 2^{2 \nu}} \int_{0}^{\infty} \frac{q^{n-1} \mathrm{~d} q}{\varpi} \frac{1}{m_{B}^{2}+p^{2}+q^{2}},
$$

which diverges as a power of the UV cutoff, $\Lambda$, as

$$
\begin{aligned}
D_{p}^{\tilde{\Lambda}}(0 ; 0) & =-\frac{i}{(\nu !)^{2} 2^{2 \nu}} \int_{0}^{\tilde{\Lambda}} \frac{q^{n-1} \mathrm{~d} q}{\varpi} \frac{1}{m_{B}^{2}+p^{2}+q^{2}} \\
& =-\frac{i}{\varpi(\nu !)^{2} 2^{2 \nu}}\left[\frac{q^{n}}{n P^{2}}-\frac{q^{n+2}}{(n+2) P^{4}}{ }_{2} F_{1}\left(1, \frac{n+2}{2} ; \frac{n+4}{2},-\frac{q^{2}}{P^{2}}\right)\right]_{0}^{\tilde{\Lambda}}
\end{aligned}
$$


where $P^{2}=m_{B}^{2}+p^{2}$ and ${ }_{2} F_{1}(a, b ; c ; z)$ denotes the Hypergeometric function.

Our focus is on even $n, n=2 m$, in which case the hypergeometric function can be simplified to the following terminating series

$$
{ }_{2} F_{1}(1, m+1, m+2, z)=-(m+1) z^{-(m+1)}\left[\log (1-z)+\sum_{j=1}^{m} \frac{z^{j}}{j}\right] .
$$

Using this in the expression of the brane-brane propagator for even codimensions, we get

$$
\begin{aligned}
D_{p}^{\tilde{\Lambda}}(0 ; 0)=\frac{i 2^{2-m}}{\varpi[\Gamma(m)]^{2}}\left[\frac{(-)^{m}}{2}\right. & P^{2(m-1)} \log \left(1+\frac{q^{2}}{P^{2}}\right) \\
& \left.+\frac{1}{2} \sum_{j=1}^{m-1} \frac{(-)^{j-m}}{j} q^{2 j} P^{2(m-1-j)}\right]_{0}^{\tilde{\Lambda}} .
\end{aligned}
$$

For even codimension, $n=2 m$, we redefine $\Lambda^{2}=\tilde{\Lambda}^{2}+P^{2}$, leading to

$$
D_{p}^{\Lambda}=-\frac{i 2^{2-m}}{\varpi[\Gamma(m)]^{2}}\left(-P^{2}\right)^{m-1}\left[\log \Lambda+\sum_{j=1}^{m-1} \frac{1}{2 j}\left(1-\frac{\Lambda^{2}}{P^{2}}\right)^{j}\right]+(\text { finite }) .
$$

For odd codimensions, a similar argument gives

$$
D_{p}^{\Lambda}=-\frac{i 2^{2-n}}{\varpi[\Gamma(n / 2)]^{2}} \sum_{j=0}^{[n / 2-1]}(-)^{j} \frac{P^{2 j} \Lambda^{n-2-2 j}}{n-2-2 j}+\text { (finite) },
$$

where $[n / 2-1]$ denotes the largest integer smaller than $n / 2-1$.

Renormalization proceeds as for codimension two, with the requirement that

$$
\frac{\lambda_{2}(\Lambda)}{1-i \lambda_{2}(\Lambda) D_{k}^{\Lambda}(0,0)}=\frac{\lambda_{2}(\mu)}{1-i \lambda_{2}(\mu) D_{k}^{\mu}(0,0)}
$$

where $\mu$ is the renormalization scale, leading to the following expression,

$$
\frac{1}{\lambda_{2}(\Lambda)}=\frac{1}{\lambda_{2}(\mu)}+i\left(D_{k}^{\Lambda}-D_{k}^{\mu}\right)
$$

The divergence of propagator on the brane also induces divergences in the expression of the 4-point function, which should be absorbed by a renormalization of $\lambda_{4}$,

$$
\begin{aligned}
G_{k_{1}, k_{2}, k_{3}, k_{4}}^{(4)}\left(y_{1} ; y_{2} ; y_{3} ; y_{4}\right) & =-6 i \lambda_{4}\left[\prod_{i=1}^{4} G_{k_{i}}^{(2)}\left(y_{i} ; 0\right)\right] \delta^{4}\left(\sum_{i} k_{i}\right) \\
& =-6 i \lambda_{4}\left[\prod_{i=1}^{4} \frac{D_{k_{i}}\left(y_{i} ; 0\right)}{1-i \lambda_{2} D_{k_{i}}(0 ; 0)}\right] \delta^{4}\left(\sum_{i} k_{i}\right)
\end{aligned}
$$

The quantity $\lambda_{4} /\left(1-i \lambda_{2} D_{k_{i}}(0,0)\right)^{4}$ is finite if $\lambda_{4}$ is renormalized in the following way

$$
\lambda_{4}(\Lambda)=\frac{\bar{\lambda}_{4}}{\left(1+i \bar{\lambda}_{2}\left(D_{k}^{\Lambda}-D_{k}^{\mu}\right)\right)^{4}} .
$$

Similar expressions can be found for higher-point couplings. 


\section{C.2 Boundary condition and energy density}

We now turn to the classical solutions for $\phi(r)$, and the boundary conditions which communicate the information of the brane potential to the bulk theory. Just as in the main text the singular form of the bulk solutions require us to regularize the boundary condition by evaluating it at $r=\epsilon$ rather than at $r=0$. Smooth results are obtained as $\epsilon \rightarrow 0$ once the bare couplings are eliminated in terms of the renormalized couplings.

The classical solution to the bulk field equation that vanishes far from the brane is

$$
\phi(r)=\bar{\phi} \frac{K_{\nu}\left(m_{B} r\right)}{\left(m_{B} r\right)^{\nu}} .
$$

Integrating the equation of motion over the brane, we obtain the boundary condition

$$
\varpi \epsilon^{n-1} \phi_{\epsilon}^{\prime}=-\lambda_{2} \phi_{\epsilon}+\lambda_{4} \phi_{\epsilon}^{3} .
$$

The energy density for such a field configuration is similarly given by

$$
\begin{aligned}
\mathcal{H} & =v \int_{\epsilon}^{\infty} r^{n-1} \mathrm{~d} r\left[\frac{1}{2}\left(\partial_{r} \phi\right)^{2}+\frac{1}{2} m_{B}^{2} \phi^{2}\right]+U_{b}(\phi(\epsilon)) \\
& =v \frac{m_{B}^{2}}{2} \bar{\phi}^{2} \epsilon^{n+1}\left(m_{B} \epsilon\right)^{-n} K_{\nu}\left(m_{B} \epsilon\right) K_{\nu+1}\left(m_{B} \epsilon\right)+U_{b}(\phi(\epsilon)) .
\end{aligned}
$$

In general both of these last equations become finite once expressed in terms of renormalized quantities, although the cancellation becomes more regularization dependent in the highercodimension case due to the appearance there of power-law divergences rather than logarithms. Rather than working this through in complete generality, we restrict ourselves here to an illustrative calculation for codimension three.

\section{C.3 Codimension-3}

For a codimension-3 brane the divergent part of the brane-brane propagator goes as

$$
D_{p}^{\Lambda}=-\frac{2 i \Lambda}{\pi \varpi}
$$

and so the divergent part of the boundary condition (C.21) cancels identically if $2 \Lambda / \pi=\epsilon$. The leading order part of the boundary condition becomes

$$
\bar{\phi}\left(m_{B}+\frac{\varpi}{\bar{\lambda}_{2}}-\mu+\frac{\pi \varpi^{3} \bar{\lambda}_{4}}{2 \bar{\lambda}_{2}^{4} m_{B}^{2}} \bar{\phi}^{2}\right)=0,
$$

where to simplify the notation we rescale $\mu \rightarrow \pi \mu / 2$. The system has solution $\bar{\phi}=0$ as well as

$$
\bar{\phi}^{2}=-\left(\frac{2 \bar{\lambda}_{2}^{4} m_{B}^{2}}{\pi \varpi^{3} \bar{\lambda}_{4}}\right) m_{\mathrm{eff}},
$$

although the second solution is only possible when

$$
m_{\mathrm{eff}}=\left(\frac{\varpi}{\bar{\lambda}_{2}}-\mu+m_{B}\right)<0 .
$$


These conclusions are consistent with the form of the energy density, which in this case is

$$
\mathcal{H}=\left(\frac{\pi \varpi}{4 m_{B}^{2}}\right) m_{\mathrm{eff}} \bar{\phi}^{2}+\frac{\bar{\lambda}_{4}}{4}\left(\frac{\varpi}{\bar{\lambda}_{2} m_{B}}\right)^{4}\left(\frac{\pi}{2}\right)^{2} \bar{\phi}^{4} .
$$

Notice that the criterion for having a nonzero v.e.v. in this case depends more strongly on $m_{B}$, relative to the codimension- 2 case.

A similar argument can be made for higher codimensions. Notice that for codimension-4 and higher, the propagator includes sub-leading divergences which should also be renormalized. Doing so, we recover a finite energy density with slightly different criteria on having a nonzero v.e.v.

\section{Bulk Goldstone modes}

A natural worry arises when the Higgs is regarded as a bulk scalar while the Standard Model gauge bosons are confined to a brane. Since the bulk $S U(2) \times U(1)$ rotations are not gauged, their spontaneous breaking might be expected to bulk Goldstone modes, corresponding to KK towers of bulk scalar modes whose lightest members are massless (or with masses set by the KK scale, if the global symmetries are broken by boundary conditions). Since only three of these 4D KK states are eaten by the Higgs mechanism, the remainder could survive and generate a potentially dangerous large number of light states. In this section, we show that only three massless Goldstone modes are produced, all of which are eaten by the gauge fields on the brane.

We start with the argument in a nutshell: when choosing a specific vacuum, such as the unitary gauge choice of the main text, one expects Goldstone modes connecting to nearby vacua. Since all vacua have the same profile in the extra dimensions, the Goldstone modes also share this profile. The modes with the smallest energy cost have only momentum along the brane directions, and so are effectively already four-dimensional. These modes turn out to be the self-localized states of those components of the Higgs doublet that do not acquire a v.e.v.

To see this explicitly we repeat the calculation of the light states in section 2.3, for the Higgs doublet $H$. The equation of motion analogous to Eq. (2.33) is

$$
\left[\frac{1}{r^{2}}\left(\left(r \partial_{r}\right)^{2}+\partial_{\theta}^{2}\right)+\partial_{\mu} \partial^{\mu}-m_{B}^{2}\right] H=\frac{\delta_{+}(r)}{2 \pi r}\left(-\lambda_{2}+\lambda_{4} H^{\star} H\right) H
$$

This equation may be linearized around the vacuum by setting

$$
H=\left(\begin{array}{c}
0 \\
\varphi(r)
\end{array}\right)+\sum_{n \geq 0}^{\infty}\left(\begin{array}{c}
\zeta_{1}^{n}(r)+i \zeta_{2}^{n}(r) \\
\chi^{n}(r)+i \zeta_{3}^{n}(r)
\end{array}\right) \sin (n \theta),
$$

with $\varphi(r)=\bar{\phi} K_{0}\left(m_{B} r\right)$ and where we introduce an infinite tower of excitation modes along the angular direction. Each one of these modes satisfies the equations of motion

$$
\left[\frac{1}{r} \partial_{r}\left(r \partial_{r}\right)-\frac{n^{2}}{r^{2}}-k^{2}\right] \chi^{n}=\frac{\delta_{+}(r)}{2 \pi r}\left(-\lambda_{2}+3 \lambda_{4} \varphi^{2}(r)\right) \chi^{n}
$$




$$
\left[\frac{1}{r} \partial_{r}\left(r \partial_{r}\right)-\frac{n^{2}}{r^{2}}-k^{2}\right] \zeta_{i}^{n}=\frac{\delta_{+}(r)}{2 \pi r}\left(-\lambda_{2}+\lambda_{4} \varphi^{2}(r)\right) \zeta_{i}^{n},
$$

where, as before, $k^{2}=m_{B}^{2}-\omega^{2}$.

The field $\chi^{0}$ is the 'physical' self-localized state, discussed in the main text, and has a mass as calculated in Eq. (2.37) with 2.38). The same goes through for the zero mode of the other fields $\zeta_{i}^{0}$, but taking into account the different factor of $\lambda_{4}$ between equations (D.3) and (D.4), their masses are given by

$$
\omega_{\zeta^{0}}^{2}=m_{B}^{2}\left[1-e^{-4 \pi / \lambda_{\zeta^{0}}}\right]
$$

with now

$$
\frac{1}{\lambda_{\zeta^{0}}}=\frac{1}{\lambda_{2 \star}}\left[1+\frac{4 \pi^{2} \bar{\lambda}_{4} \bar{\phi}^{2}}{\lambda_{2 \star}^{3}}\right]
$$

In the broken phase, $\bar{\phi}$ is given by Eq. (2.30) which leads to

$$
\frac{1}{\lambda_{\zeta^{0}}}=0
$$

showing there are three massless $4 \mathrm{D}$ Goldstone modes, $\zeta_{i}^{0}$. The bulk profile of these modes is enforced by the boundary condition imposed on the brane, and as argued in section 3.1, choosing unitary gauge on the brane removes these three massless states as they become 'eaten' by the brane gauge fields.

Turning now to the infinite tower of angular dependent modes $(n \neq 0)$, the profile of these modes is now of the form $\chi^{n}, \zeta_{i}^{n}=N_{i}^{n} K_{n}(k r)$, where $N_{i}^{n}$ is the normalization constant and we expect $k$ to be determined by the the boundary condition (2.35) which takes the form

$$
\left.2 \pi r \partial_{r}\left(\begin{array}{c}
\chi^{n}(r) \\
\zeta_{i}^{n}(r)
\end{array}\right)\right|_{\epsilon}=\left.\left(-\lambda_{2}+\left(\begin{array}{l}
3 \\
1
\end{array}\right) \lambda_{4} \varphi(r)^{2}\right)\left(\begin{array}{c}
\chi^{n} \\
\zeta_{i}^{n}
\end{array}\right)\right|_{\epsilon} .
$$

In the limit $\epsilon \rightarrow 0$, this reduces to

$$
2^{n} \pi N_{i}^{n}(k \epsilon)^{-n}\left(n !+\frac{(n-1) !}{\log \left(\epsilon m_{B} e^{\gamma} / 2\right)}\right)=\mathcal{O}\left((k \epsilon)^{-n+2}\right) .
$$

We see we must have $N_{i}^{n}=0$ if these modes are to remain bounded, and so there are therefore no light modes of this form having $\omega<m_{B}$. All the remaining excitations along the radial direction form a Kaluza-Klein tower of states starting at the bulk mass $m_{B}$ and are thus harmless. There are therefore only three massless states $\zeta_{i}^{0}$ playing the role of fourdimensional Goldstone modes, one self-localized massive mode $(\chi)$ with mass $0<m<m_{B}$ and a tower of Higgs excitations with mass higher than the bulk mass. 